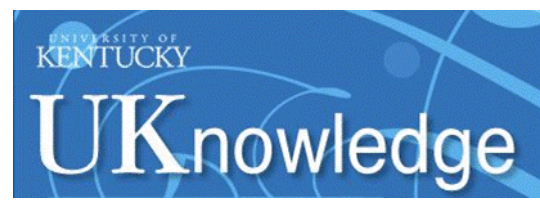

University of Kentucky

UKnowledge

7-1-1996

\title{
Atomic Data for Astrophysics. II. New Analytic FITS for Photoionization Cross Sections of Atoms and lons
}

\author{
D. A. Verner \\ University of Kentucky \\ Gary J. Ferland \\ University of Kentucky, gary@uky.edu \\ K. T. Korista \\ University of Kentucky \\ D. G. Yakovlev \\ loffe Institute of Physics and Technology, Russia
}

Follow this and additional works at: https://uknowledge.uky.edu/physastron_facpub

Part of the Astrophysics and Astronomy Commons, and the Physics Commons

Right click to open a feedback form in a new tab to let us know how this document benefits you.

\section{Repository Citation}

Verner, D. A.; Ferland, Gary J.; Korista, K. T.; and Yakovlev, D. G., "Atomic Data for Astrophysics. II. New Analytic FITS for Photoionization Cross Sections of Atoms and lons" (1996). Physics and Astronomy Faculty Publications. 153.

https://uknowledge.uky.edu/physastron_facpub/153

This Article is brought to you for free and open access by the Physics and Astronomy at UKnowledge. It has been accepted for inclusion in Physics and Astronomy Faculty Publications by an authorized administrator of UKnowledge. For more information, please contact UKnowledge@lsv.uky.edu. 


\section{Atomic Data for Astrophysics. II. New Analytic FITS for Photoionization Cross}

Sections of Atoms and lons

Digital Object Identifier (DOI)

http://dx.doi.org/10.1086/177435

Notes/Citation Information

Published in The Astrophysical Journal, v. 465, no. 1, p. 487-498.

(c) 1996. The American Astronomical Society. All rights reserved.

The copyright holder has granted permission for posting the article here. 


\title{
ATOMIC DATA FOR ASTROPHYSICS. II. NEW ANALYTIC FITS FOR PHOTOIONIZATION CROSS SECTIONS OF ATOMS AND IONS
}

\author{
D. A. Verner, ${ }^{1}$ G. J. Ferland, AND K. T. Korista \\ Department of Physics and Astronomy, University of Kentucky, Lexington, KY 40506 \\ AND \\ D. G. YAKOVLEV \\ Ioffe Institute of Physics and Technology, St. Petersburg 194021, Russia \\ Received 1995 October 27; accepted 1996 January 11
}

\begin{abstract}
We present a complete set of analytic fits to the nonrelativistic photoionization cross sections for the ground states of atoms and ions of elements from $\mathrm{H}$ through $\mathrm{Si}$, and $\mathrm{S}, \mathrm{Ar}, \mathrm{Ca}$, and $\mathrm{Fe}$. Near the ionization thresholds, the fits are based on the Opacity Project theoretical cross sections interpolated and smoothed over resonances. At higher energies, the fits reproduce calculated Hartree-Dirac-Slater photoionization cross sections.
\end{abstract}

Subject headings: atomic data - atomic processes

\section{INTRODUCTION}

Photoionization cross sections of atoms and ions are needed for many astrophysical applications. In earlier papers (Verner et al. 1993, hereafter Paper I; Verner \& Yakovlev 1995, hereafter Paper II) we presented the Hartree-Dirac-Slater (HDS) calculations and analytic fits to the partial photoionization cross sections for all subshells $n l$ of the ground states of all atoms and ions from $\mathrm{H}$ to $\mathrm{Zn}$. Comparison with available experimental and theoretical data made in Paper II demonstrated the high accuracy of the cross section fits for inner shells and far from the thresholds of outer shells. However, the theoretical HDS cross sections can be inaccurate near the thresholds of the outer shells, especially for neutral and low ionized species. The recent Opacity Project (OP) calculations (Seaton et al. 1992) based on the higher level methods such as the $R$-matrix method give more accurate low-energy photoionization cross sections.

In this paper we present a set of analytic fits to the photoionization cross sections, interpolated and smoothed over resonances, for the ground states of atoms and all ions of the OP elements $(\mathrm{H}, \mathrm{He}, \mathrm{Li}, \mathrm{Be}, \mathrm{B}, \mathrm{C}, \mathrm{N}, \mathrm{O}, \mathrm{F}, \mathrm{Ne}, \mathrm{Na}, \mathrm{Mg}$, $\mathrm{Al}, \mathrm{Si}, \mathrm{S}, \mathrm{Ar}, \mathrm{Ca}, \mathrm{Fe}$ ). Our fits are valid in the energy range from the ionization threshold up to the first cross section jump due to inner shell photoionization. In combination with the earlier fits to the inner shell cross sections (Paper II), we obtain an accurate and complete set of nonrelativistic photoionization cross sections for calculating the ionization balance in astrophysical plasmas.

\section{DATA}

We have retrieved the ground-state photoionization cross sections for all the OP species from a database TOPbase version 0.7 (Cunto et al. 1993). The OP calculations reproduce many autoionization resonances near the ionization thresholds. For many practical purposes, it is often sufficient to use the photoionization cross sections smoothed over the resonances (except for some very wide resonances). Autoionization resonances could be important for calculating the photoionization rate produced by radiation in spectral lines whose positions coincide with the

\footnotetext{
${ }^{1}$ verner@pa.uky.edu.
}

autoionization resonances. However, the OP theoretical energy levels deviate from experimental ones by $1 \%-2 \%$ (Verner, Barthel, \& Tytler 1994). This accuracy is clearly insufficient for astrophysical spectroscopy, because the uncertainty in the position is greater than the width of the resonances. If one would use the original OP photoionization cross sections for calculating the photoionization rates produced by radiation in spectral lines, one could obtain occasional coincidences and/or disagreements between spectral line energies and the OP resonances, which do not occur in nature. Therefore, smoothing of the OP photoionization cross sections is preferable.

For ions with a number of electrons $5 \leq N \leq 10$, the OP calculations give the total photoionization cross section from $2 p+2 s$ shells. The HDS calculations show a jump in the total cross section at the $2 s$ shell ionization threshold. However, more advanced $R$-matrix calculations reveal a series of autoionization resonances approaching the $2 s$ threshold. After smoothing over the resonances, the jump in the total cross section disappears. Experimental data available (see, e.g., Samson \& Angel 1990) do not show such jumps either. However, a large cross section jump at the subsequent $1 s$ ionization threshold is observable. This jump at the $1 s$ shell edge is real due to a large energy difference between the $1 s$ and $2 s$ shell ionization potentials. Therefore, it is reasonable to give a fit to the total $(2 p+2 s)$ photoionization cross section from the ionization threshold up to the $1 s$ shell edge, and to supplement it by a separate fit to the $1 s$ shell photoionization cross section at energies above the $1 \mathrm{~s}$ edge energy. Note that the inner $1 s$ shell ionization leads to subsequent ejection of Auger electrons.

A similar situation occurs for the $3 p$ and $3 s$ photoionization cross sections of the third-row elements with $13 \leq N \leq 18$. The OP data present the total cross sections, and there are no jumps at the $3 s$ ionization thresholds. For these species, we give the fits to the total $(3 p+3 s)$ cross sections. The photoionization from inner $2 p, 2 s$, and $1 s$ shells is followed by ejection of Auger electrons. The partial photoionization cross sections from these shells can be taken from Paper II; the probabilities of corresponding Auger processes are listed by Kaastra \& Mewe (1993).

The $R$-matrix OP calculations have been done at energies below the largest energy of the target states in the close- 
coupling expansion. At higher energies, the TOPbase gives simple power-law tails. Comparison with experiment made in Paper II shows that these tails are inaccurate. They have been excluded from our consideration. For each species, the low-energy OP data were interpolated, and the dataset with a constant energy step ( $\leq$ the smallest step in the original OP data) was created. This dataset was smoothed until all resonances disappeared. In most of the cases, this happened at an energy step $\Delta E / E<2 \%-3 \%$, which is comparable with the accuracy of the OP energy calibration. In some cases, very big resonances cannot be smoothed at any reasonable energy step. In these cases, our fits match the effective background determined from smoothing of all smaller resonances.

For most of the species, the smoothed OP data at the largest energies calculated by the OP are in a very good agreement with the fit to the total photoionization cross section from Paper II. Discrepancies are usually less than $2 \%$. In these cases, we simply add the HDS data to the smoothed OP data at higher energies to trace the cross section up to the subsequent inner-shell ionization edge. The situation is more complicated for neutrals and singly ionized species with outer $3 p$ electron. The HDS data do not match the OP data at the highest OP energy below the power-law tail. Experimental data for these species are available in the case of Ar I. Figure 1 shows the recommended experimental photoionization cross section of $\operatorname{Ar}$ I (Marr $\&$ West 1976). Both the experimental and the OP data show the cross section maximum near the threshold. Both the experimental and the HDS data show the cross section minimum at a higher energy, and then the second cross section maximum. The OP data are available only at energies below the position of the cross section minimum. The absolute value of the cross section minimum is in reasonable agreement with the HDS calculation, but the HDS data do not give an energy position of the minimum. The HDS data are in good agreement with the experiment at energies above the energy of the second cross section maximum. For fitting such cases, we have used the smoothed OP data supplemented by the HDS data above the second maximum. We have also taken into account the absolute value of the cross section minimum. For Ar I, we fitted the recommended experimental data. We also give a fit to the smoothed experimental data for the $4 \mathrm{~s}$ shell photoionization cross section of Ca II (Lyon et al. 1987), since the TOPbase gives only a simple power-law tail for this ion. The TOPbase does not include the data for $\mathrm{Fe}$ I and $\mathrm{Fe}$ II. For these species, we have used the smoothed total $(4 s+3 d)$ photoionization cross sections taken from the $R$ matrix calculations by Nahar \& Pradhan (1994) and Bautista \& Pradhan (1995). For He I, we give a fit to highly accurate experimental absolute photoionization cross section measured by Samson et al. (1994).

\section{FITS TO THE PHOTOIONIZATION CROSS SECTIONS}

We propose to describe the photoionization cross sections $\sigma(E)$ from the outer shells of atoms and ions in question $(\S 2)$ by the fitting formula:

$$
\begin{gathered}
\sigma(E)=\sigma_{0} F(y) \mathrm{Mb}, \quad x=\frac{E}{E_{0}}-y_{0}, \quad y=\sqrt{x^{2}+y_{1}^{2}}, \\
F(y)=\left[(x-1)^{2}+y_{\mathrm{w}}^{2}\right] y^{0.5 P-5.5}\left(1+\sqrt{y / y_{\mathrm{a}}}\right)^{-P},
\end{gathered}
$$

where $E$ is photon energy in $\mathrm{eV}$, and $\sigma_{0}, E_{0}, y_{\mathrm{w}}, y_{\mathrm{a}}, P, y_{0}$, and $y_{1}$ are the fit parameters $\left(1 \mathrm{Mb}=10^{-18} \mathrm{~cm}^{2}\right)$. Equation (1) generalizes the fitting formula used in Papers I and II, and it reduces to the formula of Papers I and II if $y_{0}=$ $y_{1}=0$. Additional fitting parameters $y_{0}$ and $y_{1}$ are required to describe adequately the more complicated behavior of the smoothed OP data near the thresholds of the outer

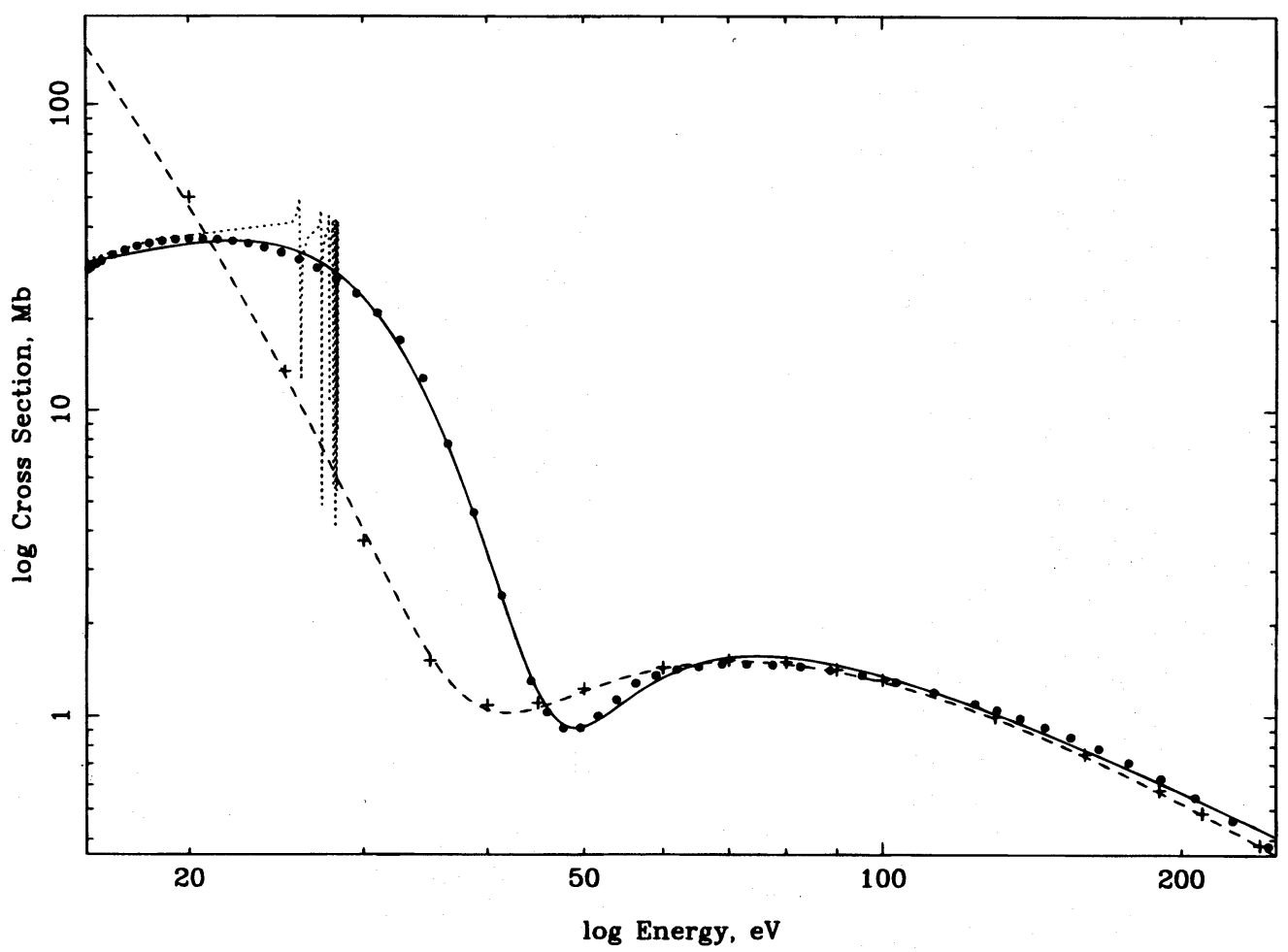

FIG. 1.-Total photoionization cross section of Ar I. Solid line, present work; dashed line, fit from Paper II; dotted line, Opacity Project data; circles, recommended experimental data from Marr \& West (1976); crosses, calculations from Reilman \& Manson (1979). 
TABLE 1

Fit Parameters for Photolonization Cross Sections ${ }^{\mathrm{a}}$

\begin{tabular}{|c|c|c|c|c|c|c|c|c|c|c|c|c|}
\hline Ion & $Z$ & $N$ & $\begin{array}{c}E_{\text {th }} \\
(\mathrm{eVV})\end{array}$ & $\begin{array}{l}E_{\max } \\
(\mathrm{eV})\end{array}$ & $\begin{array}{c}E_{0} \\
(\mathrm{eV})\end{array}$ & $\begin{array}{c}\sigma_{0} \\
(\mathrm{Mb})\end{array}$ & $y_{\mathrm{a}}$ & $P$ & $y_{\mathrm{w}}$ & $y_{0}$ & $y_{1}$ & Notes \\
\hline & 1 & 1 & $1.360 \mathrm{E}+1$ & $5.000 \mathrm{E}+4$ & $4.298 \mathrm{E}-1$ & $5.475 \mathrm{E}+4$ & $3.288 \mathrm{E}+1$ & $2.963 \mathrm{E}+0$ & $0.000 \mathrm{E}+0$ & $0.000 \mathrm{E}+0$ & $0.000 \mathrm{E}+0$ & \\
\hline He I. & 2 & 2 & $2.459 \mathrm{E}+1$ & $000 \mathrm{E}+4$ & $361 \mathrm{E}+1$ & $9.492 \mathrm{E}+2$ & $1.469 \mathrm{E}+0$ & $3.188 \mathrm{E}+0$ & & & & \\
\hline He II & 2 & 1 & $5.442 \mathrm{E}+1$ & $000 \mathrm{E}+4$ & $720 \mathrm{E}+0$ & $1.369 \mathrm{E}+4$ & $288 \mathrm{E}+1$ & $2.963 \mathrm{E}+0$ & $0.000 \mathrm{E}+0$ & $0.000 \mathrm{E}+0$ & $0.000 \mathrm{E}+0$ & A \\
\hline Li I... & 3 & 3 & $5.392 \mathrm{E}+0$ & $6.439 \mathrm{E}+1$ & $107 \mathrm{E}+0$ & $6.245 \mathrm{E}+1$ & $1.501 \mathrm{E}+1$ & $4.895 \mathrm{E}+0$ & $0.000 \mathrm{E}+0$ & $0.000 \mathrm{E}+0$ & $0.000 \mathrm{E}+0$ & \\
\hline Li II . & 3 & 2 & $7.564 \mathrm{E}+1$ & $5.000 \mathrm{E}+4$ & $2.006 \mathrm{E}+1$ & $3.201 \mathrm{E}+2$ & $7.391 \mathrm{E}+0$ & $2.916 \mathrm{E}+0$ & $0.000 \mathrm{E}+0$ & $0.000 \mathrm{E}+0$ & $0.000 \mathrm{E}+0$ & B \\
\hline Li III & 3 & 1 & $1.225 \mathrm{E}+2$ & $5.000 \mathrm{E}+4$ & $3.871 \mathrm{E}+0$ & $6.083 \mathrm{E}+3$ & $3.288 \mathrm{E}+1$ & $2.963 \mathrm{E}+0$ & $0.000 \mathrm{E}+0$ & $0.000 \mathrm{E}+0$ & $0.000 \mathrm{E}+0$ & A \\
\hline Be I. & 4 & 4 & $9.323 \mathrm{E}+0$ & $1.193 \mathrm{E}+2$ & $539 \mathrm{E}+0$ & $2.932 \mathrm{E}+5$ & $4.301 \mathrm{E}-1$ & $1.052 \mathrm{E}+1$ & $3.655 \mathrm{E}-1$ & $8.278 \mathrm{E}-4$ & $1.269 \mathrm{E}-2$ & \\
\hline $\mathrm{Be}$ II . & 4 & 3 & $1.821 \mathrm{E}+1$ & $1.299 \mathrm{E}+2$ & $1.181 \mathrm{E}+0$ & $2.678 \mathrm{E}+2$ & $5.645 \mathrm{E}+0$ & $1.170 \mathrm{E}+1$ & $0.000 \mathrm{E}+0$ & $0.000 \mathrm{E}+0$ & $0.000 \mathrm{E}+0$ & \\
\hline Be III.. & 4 & 2 & $1.539 \mathrm{E}+2$ & $5.000 \mathrm{E}+4$ & $1.760 \mathrm{E}+1$ & $5.458 \mathrm{E}+2$ & $1.719 \mathrm{E}+1$ & $3.157 \mathrm{E}+0$ & $0.000 \mathrm{E}+0$ & $0.000 \mathrm{E}+0$ & $0.000 \mathrm{E}+0$ & B \\
\hline Be IV...... & 4 & 1 & $2.177 \mathrm{E}+2$ & $5.000 \mathrm{E}+4$ & $6.879 \mathrm{E}+0$ & $3.422 \mathrm{E}+3$ & $3.288 \mathrm{E}+1$ & $2.963 \mathrm{E}+0$ & $0.000 \mathrm{E}+0$ & & $000 \mathrm{E}+0$ & A \\
\hline & 5 & 5 & $8.298 \mathrm{E}+0$ & $1.940 \mathrm{E}+2$ & $213 \mathrm{E}-1$ & $5.466 \mathrm{E}+0$ & $8.618 \mathrm{E}+0$ & $1.728 \mathrm{E}+1$ & $1.887 \mathrm{E}+1$ & $1.319 \mathrm{E}+1$ & $4.556 \mathrm{E}+0$ & \\
\hline В II... & 5 & 4 & $2.516 \mathrm{E}+1$ & $2.098 \mathrm{E}+2$ & $2.869 \mathrm{E}+0$ & $1.859 \mathrm{E}+4$ & $1.783 \mathrm{E}+0$ & $1.618 \mathrm{E}+1$ & $3.503 \mathrm{E}+0$ & $4.960 \mathrm{E}-3$ & $3.400 \mathrm{E}-2$ & \\
\hline B III... & 5 & 3 & $3.793 \mathrm{E}+1$ & $2.274 \mathrm{E}+2$ & $1.041 \mathrm{E}+0$ & $5.393 \mathrm{E}+1$ & $1.767 \mathrm{E}+1$ & $9.540 \mathrm{E}+0$ & & $0.000 \mathrm{~F}$ & +0 & B \\
\hline B IV ... & 5 & 2 & $2.594 \mathrm{E}+2$ & $5.000 \mathrm{E}+4$ & $3.336 E+1$ & $2.846 \mathrm{E}+2$ & & & & & & B \\
\hline B $\vee \ldots \ldots$ & 5 & 1 & $3.402 \mathrm{E}+2$ & $.000 \mathrm{E}+4$ & $1.075 \mathrm{E}+1$ & $2.190 \mathrm{E}+3$ & $3.288 \mathrm{E}+1$ & $2.963 \mathrm{E}+0$ & $0.000 \mathrm{E}+0$ & $0.000 \mathrm{E}+0$ & $0.000 \mathrm{E}+0$ & A \\
\hline$C_{1}{ }_{1} \ldots \ldots \ldots$. & 6 & 6 & $1.126 \mathrm{E}+1$ & $2.910 \mathrm{E}+2$ & $2.144 \mathrm{E}+0$ & $5.027 \mathrm{E}+2$ & $6.216 \mathrm{E}+1$ & $5.101 \mathrm{E}+0$ & $9.157 \mathrm{E}-2$ & $1.133 \mathrm{E}+0$ & $1.607 \mathrm{E}+0$ & \\
\hline C II ... & 6 & 5 & $2.438 \mathrm{E}+1$ & $3.076 \mathrm{E}+2$ & $4.058 \mathrm{E}-1$ & $8.709 \mathrm{E}+0$ & $1.261 \mathrm{E}+2$ & $8.578 \mathrm{E}+0$ & & & & \\
\hline C III... & 6 & 4 & $4.789 E+1$ & $3.289 \mathrm{E}+2$ & $4.614 \mathrm{E}+0$ & $1.539 E+4$ & $1.737 \mathrm{E}+0$ & & & & & $\mathrm{C}$ \\
\hline C IV... & 6 & 3 & $6.449 \mathrm{E}+1$ & $3.522 \mathrm{E}+2$ & & $1.068 \mathrm{E}+2$ & & $E+0$ & $0.000 \mathrm{E}$ & $0.000 \mathrm{E}+0$ & 80 & B \\
\hline$C v \ldots$. & 6 & 2 & $3.921 \mathrm{E}+2$ & $5.000 \mathrm{E}+4$ & $4.624 \mathrm{E}+1$ & $2.344 \mathrm{E}+2$ & $2.183 \mathrm{E}+1$ & $2.581 \mathrm{E}+0$ & $0.000 \mathrm{E}+0$ & & & B \\
\hline C VI...... & 6 & 1 & $4.900 \mathrm{E}+2$ & $5.000 \mathrm{E}+4$ & $1.548 \mathrm{E}+1$ & $1.521 \mathrm{E}+3$ & $3.288 \mathrm{E}+1$ & & & & & \\
\hline $\mathrm{N} I \ldots$. & 7 & 7 & $\mathrm{E}+1$ & $4.048 \mathrm{E}+2$ & & & & & & & & $\mathrm{C}$ \\
\hline & 7 & 6 & $\mathrm{E}+1$ & 4.23 & $E-2$ & $1.944 \mathrm{E}+0$ & $3 E+2$ & $E+0$ & $1.043 \mathrm{E}+1$ & $4.280 \mathrm{E}+2$ & & \\
\hline $\mathrm{N}$ III . & 7 & 5 & $4.745 \mathrm{E}+1$ & $4.473 \mathrm{E}+2$ & $2.420 \mathrm{E}-1$ & $9.375 \mathrm{E}-1$ & $2.788 \mathrm{E}+2$ & $9.156 \mathrm{E}+0$ & & & & $\mathrm{C}$ \\
\hline $\mathrm{N}$ IV .. & 7 & 4 & $7.747 \mathrm{E}+1$ & $4.753 E+2$ & $5.494 \mathrm{E}+0$ & $1.690 \mathrm{E}+4$ & $4 \mathrm{E}+0$ & & & & & \\
\hline $\mathrm{N}$ v ... & 7 & 3 & $9.789 \mathrm{E}+1$ & & & & & & & & & B \\
\hline $\mathrm{N}$ VI .. & 7 & 2 & & & $43 \mathrm{E}+1$ & $1.519 \mathrm{E}+2$ & $2.627 \mathrm{E}+1$ & & $0.000 \mathrm{E}+0$ & & & B \\
\hline $\mathrm{N}$ VII.. & 7 & 1 & $6.671 \mathrm{E}+2$ & $5.000 \mathrm{E}+4$ & $108 \mathrm{E}+1$ & $1.117 \mathrm{E}+3$ & $3 E+1$ & $2.963 \mathrm{E}$ & & & & A \\
\hline $\mathrm{O}_{1} \ldots$ & 8 & 8 & $2 \mathrm{E}+1$ & $5.380 \mathrm{E}+2$ & $240 \mathrm{E}+0$ & & & & & & & \\
\hline $\mathrm{O}$ II . & 8 & 7 & & & & & & & & & & $\mathrm{C}$ \\
\hline $\mathrm{O}$ III & 8 & 6 & & & & & & & & & & $\mathrm{C}$ \\
\hline $\mathrm{O}$ IV & 8 & 5 & $741 \mathrm{E}+1$ & $144 \mathrm{E}+2$ & $044 E-1$ & $659 \mathrm{E}-1$ & +2 & & & & & $\mathrm{C}$ \\
\hline $\mathrm{O} v$ & 8 & 4 & $9 \mathrm{E}+2$ & $491 \mathrm{E}+2$ & $854 \mathrm{E}+0$ & & & & & & & \\
\hline $\mathrm{O}$ VI & 8 & 3 & & & & & & & & & & B \\
\hline $\mathrm{O}$ VII... & 8 & 2 & & & & & & & & & -0 & B \\
\hline O vill .. & 8 & 1 & $4 E+2$ & & $E+1$ & & & & & & & A \\
\hline F I $\ldots$ & 9 & 9 & & & & & & & & & & \\
\hline F II.. & 9 & 8 & & & & & & & & & & $\mathrm{C}$ \\
\hline & 9 & 7 & & & & & & & & & & $\mathrm{C}$ \\
\hline$F$ IV ...... & 9 & 6 & & & $E-1$ & & & & & & & $\mathrm{C}$ \\
\hline F v & 9 & 5 & & & & & & & & & & $\mathrm{C}$ \\
\hline F VI. & 9 & 4 & & & & & & & & & & $\mathrm{C}$ \\
\hline F VII . & 9 & 3 & & & & & & & & & & B \\
\hline F VIII. & 9 & 2 & & & & & & & & & & B \\
\hline F Ix.. & & 1 & & & & & & & & & & \\
\hline Ne I.. & & 10 & & & & & & & & & & $\mathrm{C}$ \\
\hline $\mathrm{Ne}$ II . & & 9 & & & & & & & & & & $\mathrm{C}$ \\
\hline $\mathrm{Ne}$ III & & 8 & & & & & & & & & & $\mathrm{C}$ \\
\hline & & 7 & & & & & & & & & & $\mathrm{C}$ \\
\hline & & 6 & & & & & & & & & & $\mathrm{C}$ \\
\hline $\mathrm{Ne} v$ & & 5 & & & & & & & & & & $\mathrm{C}$ \\
\hline & & 4 & & & & & & & & & & $\mathrm{C}$ \\
\hline $\mathrm{Ne}$ & & 3 & & & & & & & & & & B \\
\hline $\mathrm{Ne} \mathrm{I}$ & & 2 & & & & & & & & & & B \\
\hline $\mathrm{Ne} x \ldots \ldots$ & & 1 & & & & & & & & & & A \\
\hline & & 11 & & & & & & & & & & B \\
\hline & & 10 & & & & & & & & & & $\mathrm{C}$ \\
\hline $\mathrm{Na}$ & & 9 & & & & & & & & & & $\mathrm{C}$ \\
\hline & 1 & 8 & & & & & & & & & & $\mathrm{C}$ \\
\hline & 1 & 7 & & & & & & & & & & $\mathrm{C}$ \\
\hline & & 6 & & & & & & & & & & $\mathrm{C}$ \\
\hline & & 5 & & & & & & & & & & $\mathrm{C}^{\mathrm{d}}$ \\
\hline Na VIII... & & 4 & & & & & & & & & & \\
\hline & & 3 & & & & & & & & & & B \\
\hline & & 2 & & & & & & & & & & B \\
\hline $\mathrm{Nax}$ & & 1 & & & $211 \mathrm{E}+1$ & & & & & & & \\
\hline & 12 & 12 & & & & & & & & & & \\
\hline & 1 & & & & & & & & & & & \\
\hline & 12 & 10 & & & & & & & & & $3.722 \mathrm{E}+0$ & \\
\hline $\operatorname{Mg} I$ & 12 & 9 & $1.093 E+2$ & $1.356 \mathrm{E}+3$ & $2.912 \mathrm{E}+1$ & $1.394 \mathrm{E}+3$ & $2.895 \mathrm{E}$ & $6.487 \mathrm{E}+0$ & $4.326 \mathrm{E}-2$ & $9.402 \mathrm{E}-1$ & $1.135 \mathrm{E}-1$ & $\mathrm{C}^{\mathrm{d}}$ \\
\hline $\operatorname{Mg} \vee \ldots \ldots$. & 12 & 8 & $1.413 E+2$ & $1.400 \mathrm{E}+3$ & $9.762 \mathrm{E}-1$ & $1.728 \mathrm{E}+0$ & $9.184 \mathrm{E}+1$ & $1.006 \mathrm{E}+1$ & $8.090 \mathrm{E}-1$ & $1.276 \mathrm{E}+2$ & $3.979 \mathrm{E}+0$ & \\
\hline
\end{tabular}


TABLE 1-Continued

\begin{tabular}{|c|c|c|c|c|c|c|c|c|c|c|c|c|}
\hline Ion & $Z$ & $N$ & $\begin{array}{c}E_{\mathrm{th}} \\
(\mathrm{eV})\end{array}$ & $\begin{array}{l}E_{\max } \\
(\mathrm{eV})\end{array}$ & $\begin{array}{c}E_{0} \\
(\mathrm{eV})\end{array}$ & $\begin{array}{c}\sigma_{0} \\
(\mathrm{Mb})\end{array}$ & $y_{\mathrm{a}}$ & $P$ & $y_{\mathrm{w}}$ & $y_{0}$ & $y_{1}$ & Notes \\
\hline Mg VI . & 12 & 7 & $1.865 \mathrm{E}+2$ & $1.449 \mathrm{E}+3$ & $1.711 \mathrm{E}+0$ & $2.185 \mathrm{E}+0$ & $9.350 \mathrm{E}+1$ & $9.202 \mathrm{E}+0$ & $6.325 \mathrm{E}-1$ & $1.007 \mathrm{E}+2$ & $1.729 \mathrm{E}+0$ & $\mathrm{C}$ \\
\hline Mg VII ........ & 12 & 6 & $2.249 E+2$ & $1.501 \mathrm{E}+3$ & $3.570 \mathrm{E}+0$ & $3.104 E+0$ & $6.060 \mathrm{E}+1$ & $8.857 \mathrm{E}+0$ & $1.422 \mathrm{E}+0$ & $5.452 \mathrm{E}+1$ & $2.078 \mathrm{E}+0$ & $\mathrm{C}$ \\
\hline Mg VIII $\ldots \ldots$ & 12 & 5 & $2.660 \mathrm{E}+2$ & $1.558 \mathrm{E}+3$ & $4.884 \mathrm{E}-1$ & $6.344 \mathrm{E}-2$ & $5.085 \mathrm{E}+2$ & $9.385 \mathrm{E}+0$ & $6.666 \mathrm{E}-1$ & $5.348 \mathrm{E}+2$ & $3.997 \mathrm{E}-3$ & $\mathrm{C}$ \\
\hline $\mathrm{Mg}$ IX $\ldots \ldots$ & 12 & 4 & $3.282 \mathrm{E}+2$ & $1.618 \mathrm{E}+3$ & $3.482 \mathrm{E}+1$ & $9.008 \mathrm{E}+2$ & $1.823 \mathrm{E}+0$ & $1.444 \mathrm{E}+1$ & $2.751 \mathrm{E}+0$ & $5.444 \mathrm{E}+0$ & $7.918 \mathrm{E}-2$ & C \\
\hline $\operatorname{Mg} \times \ldots \ldots \ldots$ & 12 & 3 & $3.675 \mathrm{E}+2$ & $1.675 E+3$ & $1.452 \mathrm{E}+1$ & 4.427E+1 & $3.826 \mathrm{E}+1$ & $5.460 \mathrm{E}+0$ & $0.000 \mathrm{E}+0$ & $0.000 \mathrm{E}+0$ & $0.000 \mathrm{E}+0$ & B \\
\hline Mg XI. & 12 & 2 & $1.762 \mathrm{E}+3$ & $5.000 \mathrm{E}+4$ & $2.042 E+2$ & $6.140 \mathrm{E}+1$ & $2.778 \mathrm{E}+1$ & $2.161 \mathrm{E}+0$ & $0.000 \mathrm{E}+0$ & $0.000 \mathrm{E}+0$ & $0.000 \mathrm{E}+0$ & B \\
\hline Mg XII .. & 12 & 1 & $1.963 \mathrm{E}+3$ & $5.000 \mathrm{E}+4$ & $6.203 \mathrm{E}+1$ & $3.802 \mathrm{E}+2$ & $3.288 \mathrm{E}+1$ & $2.963 \mathrm{E}+0$ & $0.000 \mathrm{E}+0$ & $0.000 \mathrm{E}+0$ & $0.000 \mathrm{E}+0$ & A \\
\hline $\mathrm{Al}$ I $\ldots \ldots \ldots$ & 13 & 13 & $5.986 \mathrm{E}+0$ & $8.040 \mathrm{E}+1$ & $1.381 \mathrm{E}+1$ & $7.195 \mathrm{E}+0$ & $1.621 \mathrm{E}+3$ & $3.642 \mathrm{E}+0$ & $3.166 \mathrm{E}-1$ & $2.041 \mathrm{E}-1$ & $4.753 \mathrm{E}-1$ & $\mathrm{E}$ \\
\hline $\mathrm{Al}$ II $\ldots \ldots \ldots$ & 13 & 12 & $1.883 \mathrm{E}+1$ & $8.997 \mathrm{E}+1$ & $2.048 \mathrm{E}-1$ & $6.948 \mathrm{E}-2$ & $5.675 E+2$ & $9.049 E+0$ & $4.615 \mathrm{E}-1$ & $9.149 E+1$ & $6.565 \mathrm{E}-1$ & $\mathrm{C}$ \\
\hline $\mathrm{Al}$ III .... & 13 & 11 & $2.845 \mathrm{E}+1$ & $1.026 \mathrm{E}+2$ & $1.027 \mathrm{E}+1$ & $4.915 \mathrm{E}+0$ & $1.990 \mathrm{E}+6$ & $3.477 \mathrm{E}+0$ & $0.000 \mathrm{E}+0$ & $0.000 \mathrm{E}+0$ & $0.000 \mathrm{E}+0$ & B \\
\hline Al IV ... & 13 & 10 & $1.200 \mathrm{E}+2$ & $1.588 \mathrm{E}+3$ & $3.130 \mathrm{E}+0$ & $1.513 E+1$ & $1.674 \mathrm{E}+1$ & $1.180 \mathrm{E}+1$ & $5.342 \mathrm{E}+0$ & $3.994 \mathrm{E}+1$ & $4.803 \mathrm{E}+0$ & $\mathrm{C}$ \\
\hline $\mathrm{Al} \mathbf{v} \ldots \ldots \ldots$ & 13 & 9 & $1.538 \mathrm{E}+2$ & $1.634 \mathrm{E}+3$ & $2.414 \mathrm{E}+1$ & $2.925 \mathrm{E}+2$ & $6.973 E+0$ & $6.724 \mathrm{E}+0$ & $1.000 \mathrm{E}-1$ & $3.495 \mathrm{E}+0$ & $2.701 \mathrm{E}-1$ & $\mathrm{C}$ \\
\hline $\mathrm{Al}$ VI .......... & 13 & 8 & $1.905 E+2$ & $1.688 \mathrm{E}+3$ & $3.483 \mathrm{E}-1$ & $1.962 \mathrm{E}-2$ & $1.856 \mathrm{E}+1$ & $2.084 \mathrm{E}+1$ & $8.839 E+0$ & $5.675 \mathrm{E}-2$ & $2.768 \mathrm{E}-1$ & $\mathrm{C}$ \\
\hline $\mathrm{Al}$ VII $\ldots \ldots \ldots$ & 13 & 7 & $2.414 \mathrm{E}+2$ & $1.739 \mathrm{E}+3$ & $2.636 \mathrm{E}+0$ & $1.889 \mathrm{E}+2$ & $1.338 \mathrm{E}+2$ & $6.204 \mathrm{E}+0$ & $1.836 \mathrm{E}+0$ & $3.552 \mathrm{E}+1$ & $8.223 \mathrm{E}-3$ & $\mathrm{C}$ \\
\hline Al VIII ........ & 13 & 6 & $2.846 \mathrm{E}+2$ & $1.799 E+3$ & $4.866 \mathrm{E}-1$ & $2.350 \mathrm{E}-1$ & $7.216 \mathrm{E}+2$ & $8.659 \mathrm{E}+0$ & $2.773 \mathrm{E}-1$ & $5.704 \mathrm{E}+2$ & $1.580 \mathrm{E}-1$ & $\mathrm{C}$ \\
\hline $\mathrm{Al} \times \ldots$ & 13 & 5 & $3.301 \mathrm{E}+2$ & $1.862 E+3$ & $1.842 \mathrm{E}+0$ & $4.982 \mathrm{E}-1$ & $2.568 \mathrm{E}+2$ & $8.406 \mathrm{E}+0$ & $6.945 \mathrm{E}-1$ & $1.719 E+2$ & $6.595 \mathrm{E}+0$ & $\mathrm{C}$ \\
\hline $\mathrm{Al} \times \ldots \ldots \ldots$ & 13 & 4 & $3.994 \mathrm{E}+2$ & $1.929 E+3$ & $8.044 \mathrm{E}+0$ & $1.774 \mathrm{E}+4$ & $1.653 \mathrm{E}+0$ & $2.655 \mathrm{E}+1$ & $2.953 E+1$ & $2.538 \mathrm{E}-2$ & $1.203 E-2$ & $\mathrm{C}$ \\
\hline $\mathrm{Al}$ xI $\ldots \ldots \ldots$ & 13 & 3 & $4.421 E+2$ & $1.992 \mathrm{E}+3$ & $2.355 E+1$ & $3.388 \mathrm{E}+1$ & $3.432 E+1$ & $5.085 \mathrm{E}+0$ & $0.000 \mathrm{E}+0$ & $0.000 \mathrm{E}+0$ & $0.000 \mathrm{E}+0$ & B \\
\hline Al XII ... & 13 & 2 & $2.086 \mathrm{E}+3$ & $5.000 \mathrm{E}+4$ & $2.738 \mathrm{E}+2$ & $4.036 \mathrm{E}+1$ & $3.567 \mathrm{E}+1$ & $1.915 \mathrm{E}+0$ & $0.000 \mathrm{E}+0$ & $0.000 \mathrm{E}+0$ & $0.000 \mathrm{E}+0$ & B \\
\hline $\mathrm{Al} \mathrm{xIII}$ & 13 & 1 & $2.304 E+3$ & $5.000 \mathrm{E}+4$ & $7.281 E+1$ & $3.239 E+2$ & $3.288 \mathrm{E}+1$ & $2.963 \mathrm{E}+0$ & $0.000 \mathrm{E}+0$ & $0.000 \mathrm{E}+0$ & $0.000 \mathrm{E}+0$ & A \\
\hline $\operatorname{Si} \mathbf{1} \ldots .$. & 14 & 14 & $8.152 \mathrm{E}+0$ & $1.060 \mathrm{E}+2$ & $2.317 \mathrm{E}+1$ & $2.506 \mathrm{E}+1$ & $2.057 \mathrm{E}+1$ & $3.546 \mathrm{E}+0$ & $2.837 \mathrm{E}-1$ & $1.672 \mathrm{E}-5$ & $4.207 \mathrm{E}-1$ & $\mathrm{E}$ \\
\hline Si II .......... & 14 & 13 & $1.635 \mathrm{E}+1$ & $1.186 \mathrm{E}+2$ & $2.556 \mathrm{E}+0$ & $4.140 \mathrm{E}+0$ & $1.337 \mathrm{E}+1$ & $1.191 \mathrm{E}+1$ & $1.570 \mathrm{E}+0$ & $6.634 \mathrm{E}+0$ & $1.272 \mathrm{E}-1$ & D \\
\hline Si III $\ldots \ldots \ldots$ & 14 & 12 & $3.349 \mathrm{E}+1$ & $1.311 E+2$ & $1.659 \mathrm{E}-1$ & $5.790 \mathrm{E}-4$ & $1.474 \mathrm{E}+2$ & $1.336 \mathrm{E}+1$ & $8.626 \mathrm{E}-1$ & $9.613 E+1$ & $6.442 \mathrm{E}-1$ & $\mathrm{C}$ \\
\hline Si IV ......... & 14 & 11 & $4.514 \mathrm{E}+1$ & $1.466 \mathrm{E}+2$ & $1.288 \mathrm{E}+1$ & $6.083 \mathrm{E}+0$ & $1.356 \mathrm{E}+6$ & $3.353 \mathrm{E}+0$ & $0.000 \mathrm{E}+0$ & $0.000 \mathrm{E}+0$ & $0.000 \mathrm{E}+0$ & B \\
\hline Si $v \ldots .$. & 14 & 10 & $1.668 \mathrm{E}+2$ & $1.887 \mathrm{E}+3$ & $7.761 \mathrm{E}-1$ & $8.863 \mathrm{E}-1$ & $1.541 \mathrm{E}+2$ & $9.980 \mathrm{E}+0$ & $1.303 E+0$ & $2.009 \mathrm{E}+2$ & $4.537 \mathrm{E}+0$ & $\mathrm{C}$ \\
\hline Si vi ........ & 14 & 9 & $2.051 \mathrm{E}+2$ & $1.946 \mathrm{E}+3$ & $6.305 \mathrm{E}+1$ & $7.293 E+1$ & $1.558 \mathrm{E}+2$ & $2.400 \mathrm{E}+0$ & $2.989 \mathrm{E}-3$ & $1.115 \mathrm{E}+0$ & $8.051 \mathrm{E}-2$ & $\mathrm{C}$ \\
\hline Si vil......... & 14 & 8 & $2.465 \mathrm{E}+2$ & $2.001 \mathrm{E}+3$ & $3.277 \mathrm{E}-1$ & $6.680 \mathrm{E}-2$ & $4.132 \mathrm{E}+1$ & $1.606 \mathrm{E}+1$ & $3.280 \mathrm{E}+0$ & $1.149 \mathrm{E}-2$ & $6.396 \mathrm{E}-1$ & $\mathrm{C}$ \\
\hline Si VIII .. & 14 & 7 & $3.032 \mathrm{E}+2$ & $2.058 \mathrm{E}+3$ & $7.655 \mathrm{E}-1$ & $3.477 \mathrm{E}-1$ & $3.733 E+2$ & $8.986 \mathrm{E}+0$ & $1.476 \mathrm{E}-3$ & $3.850 \mathrm{E}+2$ & $8.999 \mathrm{E}-2$ & $\mathrm{C}$ \\
\hline Si Ix .... & 14 & 6 & $3.511 \mathrm{E}+2$ & $2.125 E+3$ & $3.343 \mathrm{E}-1$ & $1.465 \mathrm{E}-1$ & $1.404 \mathrm{E}+3$ & $8.503 E+0$ & $1.646 \mathrm{E}+0$ & $1.036 \mathrm{E}+3$ & $2.936 \mathrm{E}-1$ & $\mathrm{C}$ \\
\hline Si $\times$.. & 14 & 5 & $4.014 \mathrm{E}+2$ & $2.194 \mathrm{E}+3$ & $8.787 \mathrm{E}-1$ & $1.950 \mathrm{E}-1$ & $7.461 \mathrm{E}+2$ & $8.302 \mathrm{E}+0$ & $4.489 \mathrm{E}-1$ & $4.528 \mathrm{E}+2$ & $1.015 \mathrm{E}+0$ & $\mathrm{C}$ \\
\hline Si XI ......... & 14 & 4 & $4.761 E+2$ & $2.268 \mathrm{E}+3$ & $1.205 \mathrm{E}+1$ & $1.992 \mathrm{E}+4$ & $1.582 \mathrm{E}+0$ & $2.425 \mathrm{E}+1$ & $2.392 \mathrm{E}+1$ & $1.990 \mathrm{E}-2$ & $1.007 \mathrm{E}-2$ & C \\
\hline Si XII $\ldots \ldots \ldots$. & 14 & 3 & $5.235 \mathrm{E}+2$ & $2.336 \mathrm{E}+3$ & $3.560 \mathrm{E}+1$ & $2.539 E+1$ & $3.307 \mathrm{E}+1$ & $4.728 \mathrm{E}+0$ & $0.000 \mathrm{E}+0$ & $0.000 \mathrm{E}+0$ & $0.000 \mathrm{E}+0$ & B \\
\hline Si XIII ......... & 14 & 2 & $2.438 \mathrm{E}+3$ & $5.000 \mathrm{E}+4$ & $2.752 \mathrm{E}+2$ & $4.754 \mathrm{E}+1$ & $2.848 \mathrm{E}+1$ & $2.135 \mathrm{E}+0$ & $0.000 \mathrm{E}+0$ & $0.000 \mathrm{E}+0$ & $0.000 \mathrm{E}+0$ & B \\
\hline Si xIV ......... & 14 & 1 & $2.673 E+3$ & $5.000 \mathrm{E}+4$ & $8.447 \mathrm{E}+1$ & $2.793 E+2$ & $3.288 \mathrm{E}+1$ & $2.963 \mathrm{E}+0$ & $0.000 \mathrm{E}+0$ & $0.000 \mathrm{E}+0$ & $0.000 \mathrm{E}+0$ & $\mathrm{~A}$ \\
\hline $\mathrm{S}_{\mathrm{I}} \ldots \ldots \ldots \ldots \ldots$ & 16 & 16 & $1.036 \mathrm{E}+1$ & $1.700 \mathrm{E}+2$ & $1.808 \mathrm{E}+1$ & $4.564 \mathrm{E}+4$ & $1.000 \mathrm{E}+0$ & $1.361 \mathrm{E}+1$ & $6.385 \mathrm{E}-1$ & $9.935 \mathrm{E}-1$ & $2.486 \mathrm{E}-1$ & $\mathrm{E}$ \\
\hline S II ........... & 16 & 15 & $2.333 \mathrm{E}+1$ & $1.846 \mathrm{E}+2$ & $8.787 \mathrm{E}+0$ & $3.136 \mathrm{E}+2$ & $3.442 \mathrm{E}+0$ & $1.281 \mathrm{E}+1$ & $7.354 \mathrm{E}-1$ & $2.782 \mathrm{E}+0$ & $1.788 \mathrm{E}-1$ & $\bar{E}$ \\
\hline S III $\ldots \ldots \ldots \ldots$ & 16 & 14 & $3.483 \mathrm{E}+1$ & $1.995 \mathrm{E}+2$ & $2.027 \mathrm{E}+0$ & $6.666 \mathrm{E}+0$ & $5.454 \mathrm{E}+1$ & $8.611 \mathrm{E}+0$ & $4.109 E+0$ & $1.568 \mathrm{E}+1$ & $9.421 \mathrm{E}+0$ & D \\
\hline S IV ........... & 16 & 13 & $4.731 \mathrm{E}+1$ & $2.164 \mathrm{E}+2$ & $2.173 \mathrm{E}+0$ & $2.606 \mathrm{E}+0$ & $6.641 \mathrm{E}+1$ & $8.655 \mathrm{E}+0$ & $1.863 \mathrm{E}+0$ & $1.975 \mathrm{E}+1$ & $3.361 \mathrm{E}+0$ & $\mathrm{C}$ \\
\hline$S \mathrm{v} \ldots \ldots \ldots \ldots$ & 16 & 12 & $7.268 E+1$ & $2.350 \mathrm{E}+2$ & $1.713 \mathrm{E}-1$ & $5.072 \mathrm{E}-4$ & $1.986 \mathrm{E}+2$ & $1.307 \mathrm{E}+1$ & $7.880 \mathrm{E}-1$ & $9.424 \mathrm{E}+1$ & $6.265 \mathrm{E}-1$ & $\mathrm{C}$ \\
\hline S vi $\ldots \ldots \ldots \ldots$ & 16 & 11 & $8.805 \mathrm{E}+1$ & $2.557 \mathrm{E}+2$ & $1.413 \mathrm{E}+1$ & $9.139 \mathrm{E}+0$ & $1.656 \mathrm{E}+3$ & $3.626 \mathrm{E}+0$ & $0.000 \mathrm{E}+0$ & $0.000 \mathrm{E}+0$ & $0.000 \mathrm{E}+0$ & B \\
\hline S vil $\ldots . \ldots \ldots$ & 16 & 10 & $2.809 \mathrm{E}+2$ & $2.569 \mathrm{E}+3$ & $3.757 \mathrm{E}-1$ & $5.703 \mathrm{E}-1$ & $1.460 \mathrm{E}+2$ & $1.135 \mathrm{E}+1$ & $1.503 \mathrm{E}+0$ & $2.222 \mathrm{E}+2$ & $4.606 \mathrm{E}+0$ & $\mathrm{C}$ \\
\hline S vill ........... & 16 & 9 & $3.282 \mathrm{E}+2$ & $2.641 \mathrm{E}+3$ & $1.462 \mathrm{E}+1$ & $3.161 \mathrm{E}+1$ & $1.611 \mathrm{E}+1$ & $8.642 \mathrm{E}+0$ & $1.153 E-3$ & $1.869 \mathrm{E}+1$ & $3.037 \mathrm{E}-1$ & $\mathrm{C}$ \\
\hline $\mathbf{S} \mathrm{Ix} \ldots \ldots \ldots$ & 16 & 8 & $3.791 E+2$ & $2.705 E+3$ & $1.526 \mathrm{E}-1$ & $9.646 \mathrm{E}+3$ & $1.438 \mathrm{E}+3$ & $5.977 \mathrm{E}+0$ & $1.492 \mathrm{E}+0$ & $1.615 \mathrm{E}-3$ & $4.049 \mathrm{E}-1$ & $\mathrm{C}$ \\
\hline $\mathrm{S} \times \ldots \ldots \ldots \ldots$ & 16 & 7 & $4.471 \mathrm{E}+2$ & $2.782 \mathrm{E}+3$ & $1.040 \mathrm{E}+1$ & $5.364 \mathrm{E}+1$ & $3.641 \mathrm{E}+1$ & $7.090 \mathrm{E}+0$ & $2.310 \mathrm{E}+0$ & $1.775 \mathrm{E}+1$ & $1.663 \mathrm{E}+0$ & $\mathrm{C}$ \\
\hline S XI .......... & 16 & 6 & $5.048 \mathrm{E}+2$ & $2.859 \mathrm{E}+3$ & $6.485 \mathrm{E}+0$ & $1.275 \mathrm{E}+1$ & $6.583 E+1$ & $7.692 \mathrm{E}+0$ & $1.678 \mathrm{E}+0$ & $3.426 \mathrm{E}+1$ & $1.370 \mathrm{E}-1$ & $\mathrm{C}$ \\
\hline S XII $\ldots \ldots \ldots$ & 16 & 5 & $5.647 \mathrm{E}+2$ & $2.941 E+3$ & $2.443 E+0$ & $3.490 \mathrm{E}-1$ & $5.411 \mathrm{E}+2$ & $7.769 E+0$ & $7.033 \mathrm{E}-1$ & $2.279 E+2$ & $1.172 \mathrm{E}+0$ & $\mathrm{C}$ \\
\hline S XIII ........... & 16 & 4 & $6.517 \mathrm{E}+2$ & $3.029 \mathrm{E}+3$ & $1.474 \mathrm{E}+1$ & $2.294 \mathrm{E}+4$ & $1.529 \mathrm{E}+0$ & $2.568 \mathrm{E}+1$ & $2.738 \mathrm{E}+1$ & $2.203 \mathrm{E}-2$ & $1.073 \mathrm{E}-2$ & $\mathrm{C}$ \\
\hline S xIV .......... & 16 & 3 & $7.072 E+2$ & $3.107 \mathrm{E}+3$ & $3.310 \mathrm{E}+1$ & $2.555 \mathrm{E}+1$ & $3.821 \mathrm{E}+1$ & $5.037 \mathrm{E}+0$ & $0.000 \mathrm{E}+0$ & $0.000 \mathrm{E}+0$ & $0.000 \mathrm{E}+0$ & B \\
\hline $\mathrm{S} \mathrm{xv} \ldots$ & 16 & 2 & $3.224 \mathrm{E}+3$ & $5.000 \mathrm{E}+4$ & $4.390 \mathrm{E}+2$ & $2.453 E+1$ & $4.405 \mathrm{E}+1$ & $1.765 \mathrm{E}+0$ & $0.000 \mathrm{E}+0$ & $0.000 \mathrm{E}+0$ & $0.000 \mathrm{E}+0$ & B \\
\hline S XVI......... & 16 & 1 & $3.494 \mathrm{E}+3$ & $5.000 \mathrm{E}+4$ & $1.104 \mathrm{E}+2$ & $2.139 E+2$ & $3.288 \mathrm{E}+1$ & $2.963 \mathrm{E}+0$ & $0.000 \mathrm{E}+0$ & $0.000 \mathrm{E}+0$ & $0.000 \mathrm{E}+0$ & $\mathrm{~A}$ \\
\hline $\operatorname{Ar} \mathbf{I} \ldots \ldots \ldots$ & 18 & 18 & $1.576 \mathrm{E}+1$ & $2.492 E+2$ & $1.709 \mathrm{E}+1$ & $2.106 \mathrm{E}+1$ & $2.645 E+2$ & $4.796 \mathrm{E}+0$ & $4.185 \mathrm{E}-1$ & $1.688 \mathrm{E}+0$ & $8.943 \mathrm{E}-1$ & $D^{b}$ \\
\hline Ar II $\ldots \ldots \ldots$ & 18 & 17 & $2.763 \mathrm{E}+1$ & $2.662 \mathrm{E}+2$ & $2.494 \mathrm{E}+1$ & $2.503 E+1$ & $1.272 \mathrm{E}+2$ & $4.288 \mathrm{E}+0$ & $5.108 \mathrm{E}-1$ & $9.299 \mathrm{E}-1$ & $7.195 \mathrm{E}-1$ & D \\
\hline Ar $\mathrm{III} . . . \ldots \ldots$ & 18 & 16 & $4.074 \mathrm{E}+1$ & $2.801 \mathrm{E}+2$ & $1.417 \mathrm{E}+1$ & $3.580 \mathrm{E}+1$ & $3.776 \mathrm{E}+1$ & $5.742 \mathrm{E}+0$ & $6.316 \mathrm{E}-1$ & $2.384 \mathrm{E}+0$ & $1.794 \mathrm{E}+0$ & D \\
\hline Ar Iv .......... & 18 & 15 & $5.981 \mathrm{E}+1$ & $2.987 \mathrm{E}+2$ & $6.953 E+0$ & $2.035 \mathrm{E}+1$ & $1.400 \mathrm{E}+1$ & $9.595 \mathrm{E}+0$ & $8.842 \mathrm{E}-1$ & $7.501 \mathrm{E}+0$ & $1.806 \mathrm{E}-1$ & $\mathrm{C}$ \\
\hline $\operatorname{Ar} \mathrm{v} \ldots \ldots \ldots$ & 18 & 14 & $7.502 E+1$ & $3.200 E+2$ & $1.031 \mathrm{E}+1$ & $9.946 \mathrm{E}+0$ & $7.444 \mathrm{E}+1$ & $6.261 \mathrm{E}+0$ & $4.885 \mathrm{E}-1$ & $6.406 \mathrm{E}+0$ & $3.659 \mathrm{E}-3$ & $\mathrm{C}$ \\
\hline $\operatorname{Ar}$ VI.......... & 18 & 13 & $9.101 \mathrm{E}+1$ & $3.426 \mathrm{E}+2$ & $5.440 \mathrm{E}-1$ & $1.080 \mathrm{E}+0$ & $9.419 E+2$ & $7.582 \mathrm{E}+0$ & $1.107 \mathrm{E}+1$ & $1.700 \mathrm{E}+2$ & $1.587 \mathrm{E}+1$ & $\mathrm{C}$ \\
\hline Ar vil ......... & 18 & 12 & $1.243 E+2$ & $3.667 \mathrm{E}+2$ & $2.966 \mathrm{E}-2$ & $3.693 \mathrm{E}+0$ & $9.951 \mathrm{E}+3$ & $7.313 E+0$ & $1.363 \mathrm{E}-2$ & $4.383 \mathrm{E}-4$ & $2.513 \mathrm{E}+0$ & $\mathrm{C}$ \\
\hline Ar viII $\ldots \ldots \ldots$ & 18 & 11 & $1.435 \mathrm{E}+2$ & $3.925 \mathrm{E}+2$ & $3.884 \mathrm{E}+0$ & $3.295 \mathrm{E}+1$ & $7.082 \mathrm{E}+2$ & $4.645 \mathrm{E}+0$ & $0.000 \mathrm{E}+0$ & $0.000 \mathrm{E}+0$ & $0.000 \mathrm{E}+0$ & B \\
\hline Ar $\mathbf{x} \ldots \ldots \ldots$ & 18 & 10 & $4.225 \mathrm{E}+2$ & $3.361 \mathrm{E}+3$ & $1.926 \mathrm{E}-1$ & $8.279 \mathrm{E}-1$ & $2.392 \mathrm{E}+2$ & $1.121 \mathrm{E}+1$ & $1.434 \mathrm{E}+0$ & $3.814 \mathrm{E}+1$ & $4.649 \mathrm{E}+0$ & $\mathrm{C}$ \\
\hline Ar $x \ldots \ldots \ldots$ & 18 & 9 & $4.787 E+2$ & $3.446 \mathrm{E}+3$ & $1.040 \mathrm{E}+1$ & $8.204 E+0$ & $1.495 \mathrm{E}+1$ & $1.115 E+1$ & $9.203 E-4$ & $3.804 \mathrm{E}+1$ & $6.390 \mathrm{E}-1$ & $\mathrm{C}$ \\
\hline Ar $x I \ldots \ldots \ldots$ & 18 & 8 & $5.390 \mathrm{E}+2$ & $3.523 E+3$ & $1.257 \mathrm{E}-1$ & $1.760 \mathrm{E}+3$ & $1.579 \mathrm{E}+3$ & $6.714 \mathrm{E}+0$ & $1.975 \mathrm{E}+0$ & $3.286 \mathrm{E}-3$ & $3.226 \mathrm{E}-1$ & $\mathrm{C}$ \\
\hline Ar XII ......... & 18 & 7 & $6.183 E+2$ & $3.613 E+3$ & $5.310 \mathrm{E}+0$ & $7.018 \mathrm{E}-1$ & $1.001 E+2$ & $8.939 E+0$ & $4.987 \mathrm{E}-1$ & $1.099 \mathrm{E}+2$ & $2.202 \mathrm{E}-1$ & $\mathrm{C}$ \\
\hline Ar XIII $\ldots . . .$. & 18 & 6 & $6.861 \mathrm{E}+2$ & $3.702 \mathrm{E}+3$ & $3.209 \mathrm{E}-1$ & $2.459 \mathrm{E}-2$ & $2.285 E+3$ & $8.810 \mathrm{E}+0$ & $6.692 \mathrm{E}-1$ & $2.068 \mathrm{E}+3$ & $2.113 E+1$ & $\mathrm{C}$ \\
\hline Ar XIV ........ & 18 & 5 & $7.558 \mathrm{E}+2$ & $3.798 \mathrm{E}+3$ & $1.557 \mathrm{E}+0$ & $4.997 \mathrm{E}-2$ & $5.031 \mathrm{E}+2$ & $8.966 \mathrm{E}+0$ & $2.938 \mathrm{E}-1$ & $4.552 \mathrm{E}+2$ & $6.459 \mathrm{E}+0$ & $\mathrm{C}$ \\
\hline Ar xv $\ldots \ldots \ldots$ & 18 & 4 & $8.548 \mathrm{E}+2$ & $3.898 \mathrm{E}+3$ & $1.888 \mathrm{E}+1$ & $2.571 \mathrm{E}+4$ & $1.475 \mathrm{E}+0$ & $2.634 \mathrm{E}+1$ & $2.909 E+1$ & $2.445 \mathrm{E}-2$ & $1.054 \mathrm{E}-2$ & $\mathrm{C}$ \\
\hline Ar XVI ........ & 18 & 3 & $9.180 \mathrm{E}+2$ & $3.988 \mathrm{E}+3$ & $4.154 \mathrm{E}+1$ & $2.135 \mathrm{E}+1$ & $4.118 \mathrm{E}+1$ & $4.945 \mathrm{E}+0$ & $0.000 \mathrm{E}+0$ & $0.000 \mathrm{E}+0$ & $0.000 \mathrm{E}+0$ & B \\
\hline Ar xvII ....... & 18 & 2 & $4.121 E+3$ & $5.000 \mathrm{E}+4$ & $4.468 \mathrm{E}+2$ & $3.108 \mathrm{E}+1$ & $3.039 \mathrm{E}+1$ & $2.092 \mathrm{E}+0$ & $0.000 \mathrm{E}+0$ & $0.000 \mathrm{E}+0$ & $0.000 \mathrm{E}+0$ & B \\
\hline Ar XVIII ....... & 18 & 1 & $4.426 \mathrm{E}+3$ & $5.000 \mathrm{E}+4$ & $1.399 \mathrm{E}+2$ & $1.690 \mathrm{E}+2$ & $3.288 \mathrm{E}+1$ & $2.963 \mathrm{E}+0$ & $0.000 \mathrm{E}+0$ & $0.000 \mathrm{E}+0$ & $0.000 \mathrm{E}+0$ & $\mathrm{~A}$ \\
\hline $\mathrm{Ca} \mathrm{I}_{1} \ldots \ldots \ldots$ & 20 & 20 & $6.113 E+0$ & $3.443 E+1$ & $1.278 \mathrm{E}+1$ & $5.370 \mathrm{E}+5$ & $3.162 \mathrm{E}-1$ & $1.242 \mathrm{E}+1$ & $4.477 \mathrm{E}-1$ & $1.012 \mathrm{E}-3$ & $1.851 \mathrm{E}-2$ & $\mathrm{E}^{\mathrm{e}}$ \\
\hline $\mathrm{Ca}$ II ........... & 20 & 19 & $1.187 \mathrm{E}+1$ & $4.090 \mathrm{E}+1$ & $1.553 E+1$ & $1.064 \mathrm{E}+7$ & $7.790 \mathrm{E}-1$ & $2.130 \mathrm{E}+1$ & $6.453 \mathrm{E}-1$ & $2.161 \mathrm{E}-3$ & $6.706 \mathrm{E}-2$ & $E^{\text {be }}$ \\
\hline Ca III $\ldots . . .$. & 20 & 18 & $5.091 \mathrm{E}+1$ & $3.731 E+2$ & $2.436 \mathrm{E}+1$ & $3.815 \mathrm{E}+1$ & $2.931 E+2$ & $3.944 \mathrm{E}+0$ & $3.126 \mathrm{E}-1$ & $1.802 \mathrm{E}+0$ & $1.233 \mathrm{E}+0$ & $\mathrm{E}$ \\
\hline $\mathrm{Ca}$ IV $\ldots \ldots \ldots$ & 20 & 17 & $6.727 \mathrm{E}+1$ & $3.944 \mathrm{E}+2$ & $4.255 \mathrm{E}+0$ & $7.736 \mathrm{E}+0$ & $1.355 \mathrm{E}+1$ & $1.236 \mathrm{E}+1$ & $1.369 \mathrm{E}+0$ & $1.467 \mathrm{E}+1$ & $3.298 \mathrm{E}-2$ & $\bar{C}$ \\
\hline
\end{tabular}


TABLE 1-Continued

\begin{tabular}{|c|c|c|c|c|c|c|c|c|c|c|c|c|}
\hline Ion & $Z$ & $N$ & $\begin{array}{c}E_{\mathrm{th}} \\
(\mathrm{eV})\end{array}$ & $\begin{array}{l}E_{\max } \\
(\mathrm{eV})\end{array}$ & $\begin{array}{c}E_{0} \\
(\mathrm{eV})\end{array}$ & $\begin{array}{c}\sigma_{0} \\
(\mathbf{M b})\end{array}$ & $y_{\mathrm{a}}$ & $P$ & $y_{\mathrm{w}}$ & $y_{0}$ & $y_{1}$ & Notes \\
\hline $\mathrm{Ca} v .$. & 20 & 16 & $8.451 \mathrm{E}+1$ & $4.175 \mathrm{E}+2$ & $6.882 \mathrm{E}-1$ & $1.523 \mathrm{E}-1$ & $1.502 \mathrm{E}+2$ & $1.061 \mathrm{E}+1$ & $8.227 \mathrm{E}+0$ & $1.210 \mathrm{E}+2$ & $3.876 \mathrm{E}+0$ & $\mathrm{C}$ \\
\hline Ca vi... & 20 & 15 & $1.088 \mathrm{E}+2$ & $4.423 E+2$ & $9.515 \mathrm{E}+0$ & $7.642 \mathrm{E}+1$ & $8.973 E+1$ & $5.141 \mathrm{E}+0$ & $2.471 \mathrm{E}+0$ & $4.829 \mathrm{E}+0$ & $5.824 \mathrm{E}+0$ & $\mathrm{C}$ \\
\hline Ca VII.. & 20 & 14 & $1.272 \mathrm{E}+2$ & $4.687 \mathrm{E}+2$ & $8.080 \mathrm{E}-1$ & $4.760 \mathrm{E}-1$ & $3.682 \mathrm{E}+2$ & $8.634 \mathrm{E}+0$ & $5.720 \mathrm{E}-1$ & $1.487 \mathrm{E}+2$ & $1.283 \mathrm{E}+0$ & C \\
\hline Ca VIII ........ & 20 & 13 & $1.472 \mathrm{E}+2$ & $4.967 \mathrm{E}+2$ & $1.366 \mathrm{E}+0$ & $6.641 \mathrm{E}-1$ & $3.188 E+2$ & $8.138 \mathrm{E}+0$ & $2.806 \mathrm{E}-1$ & $1.039 \mathrm{E}+2$ & $3.329 \mathrm{E}+0$ & $\mathrm{C}$ \\
\hline $\mathrm{Ca} \mathrm{xx} . . . \ldots \ldots$ & 20 & 12 & $1.883 \mathrm{E}+2$ & $5.270 \mathrm{E}+2$ & $5.520 \mathrm{E}-2$ & $2.076 \mathrm{E}+2$ & $1.790 \mathrm{E}+4$ & $5.893 \mathrm{E}+0$ & $1.843 E-3$ & $2.826 \mathrm{E}-4$ & $1.657 \mathrm{E}+0$ & $\mathrm{C}$ \\
\hline $\mathrm{Ca} \times \ldots \ldots \ldots$ & 20 & 11 & $2.113 \mathrm{E}+2$ & $5.569 \mathrm{E}+2$ & $1.605 \mathrm{E}+1$ & $1.437 \mathrm{E}+1$ & $6.989 E+2$ & $3.857 \mathrm{E}+0$ & $0.000 \mathrm{E}+0$ & $0.000 \mathrm{E}+0$ & $0.000 \mathrm{E}+0$ & B \\
\hline $\mathrm{Ca}$ XI......... & 20 & 10 & $5.919 \mathrm{E}+2$ & $4.265 E+3$ & $2.288 \mathrm{E}-1$ & $9.384 \mathrm{E}-1$ & $2.549 \mathrm{E}+2$ & $1.103 E+1$ & $1.390 \mathrm{E}+0$ & $2.478 \mathrm{E}+1$ & $3.100 \mathrm{E}+0$ & $\mathrm{C}^{\mathrm{d}}$ \\
\hline Ca XII ... & 20 & 9 & $6.572 E+2$ & $4.362 E+3$ & $2.345 E+1$ & $1.227 \mathrm{E}+1$ & $1.312 \mathrm{E}+1$ & $9.771 \mathrm{E}+0$ & $6.842 \mathrm{E}-4$ & $2.417 \mathrm{E}+1$ & $5.469 \mathrm{E}-1$ & $\mathrm{C}^{\mathrm{d}}$ \\
\hline Ca XIII ......... & 20 & 8 & $7.267 \mathrm{E}+2$ & $4.453 \mathrm{E}+3$ & $1.008 \mathrm{E}+1$ & $1.849 \mathrm{E}+3$ & $1.792 E+4$ & $2.868 \mathrm{E}+0$ & $2.410 \mathrm{E}+2$ & $6.138 \mathrm{E}-3$ & $6.931 E+1$ & $\mathrm{C}$ \\
\hline $\mathrm{Ca}$ XIV ........ & 20 & 7 & $8.177 E+2$ & $4.555 E+3$ & $9.980 \mathrm{E}+0$ & $1.116 \mathrm{E}+0$ & $5.918 \mathrm{E}+1$ & $9.005 \mathrm{E}+0$ & $3.879 \mathrm{E}+0$ & $7.104 \mathrm{E}+1$ & $5.311 \mathrm{E}+0$ & $\mathrm{C}$ \\
\hline $\mathrm{Ca} \mathrm{xv} \mathrm{.........}$ & 20 & 6 & $8.946 \mathrm{E}+2$ & $4.659 \mathrm{E}+3$ & $1.309 \mathrm{E}+2$ & $5.513 E+1$ & $3.828 \mathrm{E}+2$ & $2.023 \mathrm{E}+0$ & $9.084 \mathrm{E}-2$ & $1.833 \mathrm{E}-2$ & $9.359 \mathrm{E}-1$ & C \\
\hline Ca XvI ........ & 20 & 5 & $9.745 E+2$ & $4.767 E+3$ & $4.293 E+0$ & $1.293 \mathrm{E}+0$ & $1.691 \mathrm{E}+1$ & $1.438 \mathrm{E}+1$ & $3.461 \mathrm{E}-5$ & $9.363 \mathrm{E}-1$ & $4.589 \mathrm{E}-2$ & $\mathrm{C}$ \\
\hline Ca XVII . & 20 & 4 & $1.087 \mathrm{E}+3$ & $4.880 \mathrm{E}+3$ & $2.618 \mathrm{E}+1$ & $2.028 E+4$ & $1.456 \mathrm{E}+0$ & $2.560 \mathrm{E}+1$ & $2.803 E+1$ & $2.402 \mathrm{E}-2$ & $9.323 \mathrm{E}-3$ & $\mathrm{C}$ \\
\hline Ca $\mathrm{xVIII} . . . . .$. & 20 & 3 & $1.157 \mathrm{E}+3$ & $4.982 \mathrm{E}+3$ & $9.472 \mathrm{E}+1$ & $1.105 \mathrm{E}+1$ & $3.818 \mathrm{E}+1$ & $4.192 \mathrm{E}+0$ & $0.000 \mathrm{E}+0$ & $0.000 \mathrm{E}+0$ & $0.000 \mathrm{E}+0$ & B \\
\hline $\mathrm{Ca} \mathrm{xIX} \ldots \ldots \ldots$ & 20 & 2 & $5.129 E+3$ & $5.000 \mathrm{E}+4$ & $6.297 E+2$ & $1.936 \mathrm{E}+1$ & $3.921 \mathrm{E}+1$ & $1.862 \mathrm{E}+0$ & $0.000 \mathrm{E}+0$ & $0.000 \mathrm{E}+0$ & $0.000 \mathrm{E}+0$ & B \\
\hline Ca Xx.. & 20 & 1 & $5.470 \mathrm{E}+3$ & $5.000 \mathrm{E}+4$ & $1.729 \mathrm{E}+2$ & $1.369 \mathrm{E}+2$ & $3.288 \mathrm{E}+1$ & $2.963 E+0$ & $0.000 \mathrm{E}+0$ & $0.000 \mathrm{E}+0$ & $0.000 \mathrm{E}+0$ & A \\
\hline $\mathrm{Fe} I \ldots$ & 26 & 26 & $7.902 \mathrm{E}+0$ & $6.600 \mathrm{E}+1$ & $5.461 \mathrm{E}-2$ & $3.062 \mathrm{E}-1$ & $2.671 E+7$ & $7.923 \mathrm{E}+0$ & $2.069 \mathrm{E}+1$ & $1.382 \mathrm{E}+2$ & $2.481 \mathrm{E}-1$ & $\mathrm{E}^{\mathrm{e}}$ \\
\hline $\mathrm{Fe}$ II .... & 26 & 25 & $1.619 \mathrm{E}+1$ & $7.617 \mathrm{E}+1$ & $1.761 \mathrm{E}-1$ & $4.365 E+3$ & $6.298 E+3$ & $5.204 \mathrm{E}+0$ & $1.141 \mathrm{E}+1$ & $9.272 \mathrm{E}+1$ & $1.075 E+2$ & $E^{e}$ \\
\hline $\mathrm{Fe}$ III $\ldots \ldots \ldots$ & 26 & 24 & $3.065 \mathrm{E}+1$ & $8.705 E+1$ & $1.698 \mathrm{E}-1$ & $6.107 \mathrm{E}+0$ & $1.555 \mathrm{E}+3$ & $8.055 \mathrm{E}+0$ & $8.698 \mathrm{E}+0$ & $1.760 \mathrm{E}+2$ & $1.847 \mathrm{E}+1$ & $D^{e}$ \\
\hline Fe IV $\ldots \ldots \ldots$ & 26 & 23 & $5.480 \mathrm{E}+1$ & $1.067 \mathrm{E}+2$ & $2.544 \mathrm{E}+1$ & $3.653 E+2$ & $8.913 E+0$ & $6.538 \mathrm{E}+0$ & $5.602 \mathrm{E}-1$ & $0.000 \mathrm{E}+0$ & $0.000 \mathrm{E}+0$ & $F^{e}$ \\
\hline $\mathrm{Fe} v \ldots \ldots \ldots$ & 26 & 22 & $7.501 \mathrm{E}+1$ & $1.288 \mathrm{E}+2$ & $7.256 \mathrm{E}-1$ & $1.523 \mathrm{E}-3$ & $3.736 \mathrm{E}+1$ & $1.767 \mathrm{E}+1$ & $5.064 \mathrm{E}+1$ & $8.871 E+1$ & $5.280 \mathrm{E}-2$ & $F^{e}$ \\
\hline Fe vi $\ldots \ldots \ldots$ & 26 & 21 & $9.906 \mathrm{E}+1$ & $1.527 \mathrm{E}+2$ & $2.656 \mathrm{E}+0$ & $5.259 \mathrm{E}-1$ & $1.450 \mathrm{E}+1$ & $1.632 \mathrm{E}+1$ & $1.558 \mathrm{E}+1$ & $3.361 \mathrm{E}+1$ & $3.743 E-3$ & $F^{e}$ \\
\hline Fe vII $\ldots \ldots \ldots$ & 26 & 20 & $1.250 \mathrm{E}+2$ & $1.783 \mathrm{E}+2$ & $5.059 \mathrm{E}+0$ & $2.420 \mathrm{E}+4$ & $4.850 \mathrm{E}+4$ & $2.374 \mathrm{E}+0$ & $2.516 \mathrm{E}-3$ & $4.546 \mathrm{E}-1$ & $2.683 E+1$ & $D^{e}$ \\
\hline $\mathrm{Fe}$ VIII $\ldots \ldots \ldots$ & 26 & 19 & $1.511 E+2$ & $2.055 E+2$ & $7.098 \mathrm{E}-2$ & $1.979 \mathrm{E}+1$ & $1.745 E+4$ & $6.750 \mathrm{E}+0$ & $2.158 \mathrm{E}+2$ & $2.542 \mathrm{E}+3$ & $4.672 \mathrm{E}+2$ & $\mathrm{D}^{\mathrm{e}}$ \\
\hline $\mathrm{Fe} \mathrm{IX} \ldots \ldots \ldots$ & 26 & 18 & $2.336 \mathrm{E}+2$ & $9.211 \mathrm{E}+2$ & $6.741 \mathrm{E}+0$ & $2.687 \mathrm{E}+1$ & $1.807 \mathrm{E}+2$ & $6.290 \mathrm{E}+0$ & $2.387 \mathrm{E}-4$ & $2.494 \mathrm{E}+1$ & $8.251 \mathrm{E}+0$ & $\mathrm{C}$ \\
\hline $\mathrm{Fe} x \ldots \ldots \ldots$ & 26 & 17 & $2.621 E+2$ & $9.590 E+2$ & $6.886 \mathrm{E}+1$ & $6.470 \mathrm{E}+1$ & $2.062 \mathrm{E}+1$ & $4.111 \mathrm{E}+0$ & $2.778 \mathrm{E}-4$ & $1.190 \mathrm{E}-5$ & $6.570 \mathrm{E}-3$ & $\mathrm{C}$ \\
\hline Fe XI $\ldots \ldots \ldots$ & 26 & 16 & $2.902 \mathrm{E}+2$ & $9.983 E+2$ & $8.284 \mathrm{E}+0$ & $3.281 \mathrm{E}+0$ & $5.360 \mathrm{E}+1$ & $8.571 \mathrm{E}+0$ & $3.279 \mathrm{E}-1$ & $2.971 \mathrm{E}+1$ & $5.220 \mathrm{E}-1$ & $\mathrm{C}$ \\
\hline $\mathrm{Fe}$ XII $\ldots \ldots \ldots$ & 26 & 15 & $3.308 \mathrm{E}+2$ & $1.039 \mathrm{E}+3$ & $6.295 \mathrm{E}+0$ & $1.738 \mathrm{E}+0$ & $1.130 \mathrm{E}+2$ & $8.037 \mathrm{E}+0$ & $3.096 \mathrm{E}-1$ & $4.671 \mathrm{E}+1$ & $1.425 \mathrm{E}-1$ & $\mathrm{C}$ \\
\hline Fe XIII $\ldots \ldots \ldots$ & 26 & 14 & $3.610 \mathrm{E}+2$ & $1.081 \mathrm{E}+3$ & $1.317 \mathrm{E}-1$ & $2.791 \mathrm{E}-3$ & $2.487 \mathrm{E}+3$ & $9.791 \mathrm{E}+0$ & $6.938 \mathrm{E}-1$ & $2.170 \mathrm{E}+3$ & $6.852 \mathrm{E}-3$ & $\mathrm{C}$ \\
\hline Fe XIV ........ & 26 & 13 & $3.922 \mathrm{E}+2$ & $1.125 \mathrm{E}+3$ & $8.509 \mathrm{E}-1$ & $1.454 \mathrm{E}-1$ & $1.239 \mathrm{E}+3$ & $8.066 \mathrm{E}+0$ & $4.937 \mathrm{E}-1$ & $4.505 \mathrm{E}+2$ & $2.504 \mathrm{E}+0$ & $\mathrm{C}$ \\
\hline $\mathrm{Fe} x \mathrm{xV} \ldots \ldots \ldots$ & 26 & 12 & $4.570 E+2$ & $1.181 E+3$ & $5.555 \mathrm{E}-2$ & $2.108 \mathrm{E}+2$ & $2.045 E+4$ & $6.033 \mathrm{E}+0$ & $1.885 \mathrm{E}-3$ & $2.706 \mathrm{E}-4$ & $1.628 \mathrm{E}+0$ & $\mathrm{C}$ \\
\hline $\mathrm{Fe}$ XVI $\ldots \ldots \ldots$ & 26 & 11 & $4.893 \mathrm{E}+2$ & $1.216 \mathrm{E}+3$ & $2.873 \mathrm{E}+1$ & $1.207 \mathrm{E}+1$ & $5.150 \mathrm{E}+2$ & $3.846 \mathrm{E}+0$ & $0.000 \mathrm{E}+0$ & $0.000 \mathrm{E}+0$ & $0.000 \mathrm{E}+0$ & B \\
\hline Fe XVII ........ & 26 & 10 & $1.262 \mathrm{E}+3$ & $7.651 \mathrm{E}+3$ & $3.444 \mathrm{E}-1$ & $1.452 \mathrm{E}+0$ & $3.960 \mathrm{E}+2$ & $1.013 \mathrm{E}+1$ & $1.264 \mathrm{E}+0$ & $2.891 \mathrm{E}+1$ & $3.404 \mathrm{E}+0$ & $\mathrm{C}$ \\
\hline Fe XVIII ....... & 26 & 9 & $1.358 \mathrm{E}+3$ & $7.769 \mathrm{E}+3$ & $3.190 \mathrm{E}+1$ & $2.388 \mathrm{E}+0$ & $2.186 \mathrm{E}+1$ & $9.589 E+0$ & $2.902 \mathrm{E}-2$ & $3.805 \mathrm{E}+1$ & $4.805 \mathrm{E}-1$ & $\mathrm{C}$ \\
\hline $\mathrm{Fe}$ XIX $\ldots \ldots \ldots$ & 26 & 8 & $1.456 \mathrm{E}+3$ & $7.918 \mathrm{E}+3$ & $7.519 \mathrm{E}-4$ & $6.066 \mathrm{E}-5$ & $1.606 \mathrm{E}+6$ & $8.813 E+0$ & $4.398 \mathrm{E}+0$ & $1.915 \mathrm{E}+6$ & $3.140 \mathrm{E}+1$ & $\mathrm{C}^{\mathrm{d}}$ \\
\hline $\mathrm{Fe} x \mathrm{x} \ldots \ldots \ldots$ & 26 & 7 & $1.582 \mathrm{E}+3$ & $8.041 E+3$ & $2.011 E+1$ & $4.455 \mathrm{E}-1$ & 4.236E +1 & $9.724 \mathrm{E}+0$ & $2.757 \mathrm{E}+0$ & $6.847 \mathrm{E}+1$ & $3.989 \mathrm{E}+0$ & $\mathrm{C}$ \\
\hline $\mathrm{Fe} \times \mathrm{XXI} \ldots \ldots$ & 26 & 6 & $1.689 E+3$ & $8.184 \mathrm{E}+3$ & $9.243 \mathrm{E}+0$ & $1.098 \mathrm{E}+1$ & $7.637 \mathrm{E}+1$ & $7.962 \mathrm{E}+0$ & $1.748 \mathrm{E}+0$ & $4.446 \mathrm{E}+1$ & $3.512 \mathrm{E}+0$ & $\mathrm{C}$ \\
\hline $\mathrm{Fe}$ XXII........ & 26 & 5 & $1.799 E+3$ & $8.350 \mathrm{E}+3$ & $9.713 E+0$ & $7.204 \mathrm{E}-2$ & $1.853 \mathrm{E}+2$ & $8.843 E+0$ & $9.551 \mathrm{E}-3$ & $1.702 \mathrm{E}+2$ & $4.263 \mathrm{E}+0$ & $\mathrm{C}$ \\
\hline Fe XXIII ....... & 26 & 4 & $1.950 \mathrm{E}+3$ & $8.484 \mathrm{E}+3$ & $4.575 \mathrm{E}+1$ & $2.580 \mathrm{E}+4$ & $1.358 \mathrm{E}+0$ & $2.604 \mathrm{E}+1$ & $2.723 E+1$ & $3.582 \mathrm{E}-2$ & $8.712 \mathrm{E}-3$ & $\mathrm{C}$ \\
\hline $\mathrm{Fe}$ XXIV ....... & 26 & 3 & $2.046 \mathrm{E}+3$ & $8.638 \mathrm{E}+3$ & $7.326 \mathrm{E}+1$ & $1.276 \mathrm{E}+1$ & $4.914 \mathrm{E}+1$ & $4.941 \mathrm{E}+0$ & $0.000 \mathrm{E}+0$ & $0.000 \mathrm{E}+0$ & $0.000 \mathrm{E}+0$ & B \\
\hline $\mathrm{Fe} x x v \ldots \ldots$ & 26 & 2 & $8.829 \mathrm{E}+3$ & $5.000 \mathrm{E}+4$ & $1.057 \mathrm{E}+3$ & $1.195 \mathrm{E}+1$ & $5.769 E+1$ & $1.718 \mathrm{E}+0$ & $0.000 \mathrm{E}+0$ & $0.000 \mathrm{E}+0$ & $0.000 \mathrm{E}+0$ & B \\
\hline Fe XXVI ....... & 26 & 1 & $9.278 \mathrm{E}+3$ & $5.000 \mathrm{E}+4$ & $2.932 \mathrm{E}+2$ & $8.099 \mathrm{E}+1$ & $3.288 \mathrm{E}+1$ & $2.963 \mathrm{E}+0$ & $0.000 \mathrm{E}+0$ & $0.000 \mathrm{E}+0$ & $0.000 \mathrm{E}+0$ & A \\
\hline
\end{tabular}

NotE.-A, B, C, D, E, F are classes of accuracy (see text).

a $1.360 \mathrm{E}+1$ denotes $1.360 \times 10^{1}$.

${ }^{b}$ Fit to experimental cross section.

${ }^{c}$ Fit goes through the averaged near-threshold resonances. A steplike structure of the near-threshold cross section is smoothed.

${ }^{d}$ In the OP cross section, there is one isolated data point that is approximately 2 orders of magnitude larger than all neighboring data (e.g., Fe XIX cross sections are $0.2143,103.8$, and $0.2421 \mathrm{Mb}$ at $1485.3,1485.5$, and $1485.6 \mathrm{eV}$, respectively). This isolated data point is not included in the averaged cross section.

${ }^{\mathrm{e}}$ Fit should not be extrapolated above $E_{\max }$

shells of neutral and low ionized species, including the twomaxima cross section structure for the third row elements.

The fit parameters $E_{0}$ and $\sigma_{0}$ determine, respectively, typical energy and cross section scales (see Papers I and II for details). Other fit parameters reflect the cross section features. In particular, $y$ can be rewritten as $y=\left\{\left[\left(E-E_{1}\right)^{2}\right.\right.$ $\left.\left.+\Gamma_{1}^{2}\right] / E_{0}\right\}^{1 / 2}$, where $E_{1}=y_{0} E_{0}$ shows the position of the first (after the threshold) cross section maximum, and $\Gamma_{1}=$ $y_{1} E_{0}$ is an "energy width" of this maximum. Similarly, $(x-1)^{2}+y_{\mathrm{w}}^{2}=\left[\left(E-E_{2}\right)^{2}+\Gamma_{2}^{2}\right] / E_{0}^{2}$, where $E_{2}=E_{1}+E_{0}$ indicates the energy position of the cross section minimum (dip), and $\Gamma_{2}=y_{\mathrm{w}} E_{0}$ can be treated as the "dip energy width." The fit parameter $P$ determines the power-law cross section behavior $\sigma(E) \propto E^{0.5 P-3.5}$ in the energy interval $E_{2} \ll E \ll E_{\mathrm{a}}$ behind the dip (provided $E_{\mathrm{a}} \gg E_{2}$ ), where $E_{\mathrm{a}}=y_{\mathrm{a}} E_{0}$ is the upper boundary of this interval. Finally, equation (1) reproduces the correct nonrelativistic high- energy asymptote, $\sigma(E) \propto E^{-3.5}$, for $E \gg E_{\text {a }}$. Note that all of the cross sections to be fitted ( $(2)$ include the contribution of the outer $s$ subshell at high $E$. If we considered the partial photoionization cross sections for another subshell $l$, equation (1) should have been modified by replacing $0.5 P-5.5$ with $0.5 P-5.5-l$ to ensure the correct high-energy asymptote (see Papers I and II).

Equation (1) can be generalized further to describe more complicated near-threshold behavior of the photoionization cross sections smoothed over resonances. For instance, the smoothed $(3 p+3 s)$-shell ionization cross section of $\mathrm{S}_{\mathrm{I}}$ looks as if another minimum occurs very near the outer $3 p$-shell ionization threshold. This conclusion is not definite, since there are many autoionization near-threshold resonances. If, however, this were so, we could include the effect into the fitting by introducing into (1) an additional factor $\left[\left(E-E_{3}\right)^{2}+\Gamma_{3}^{2}\right] / E^{2}$, where $E_{3}$ and $\Gamma_{3}$ are two new 


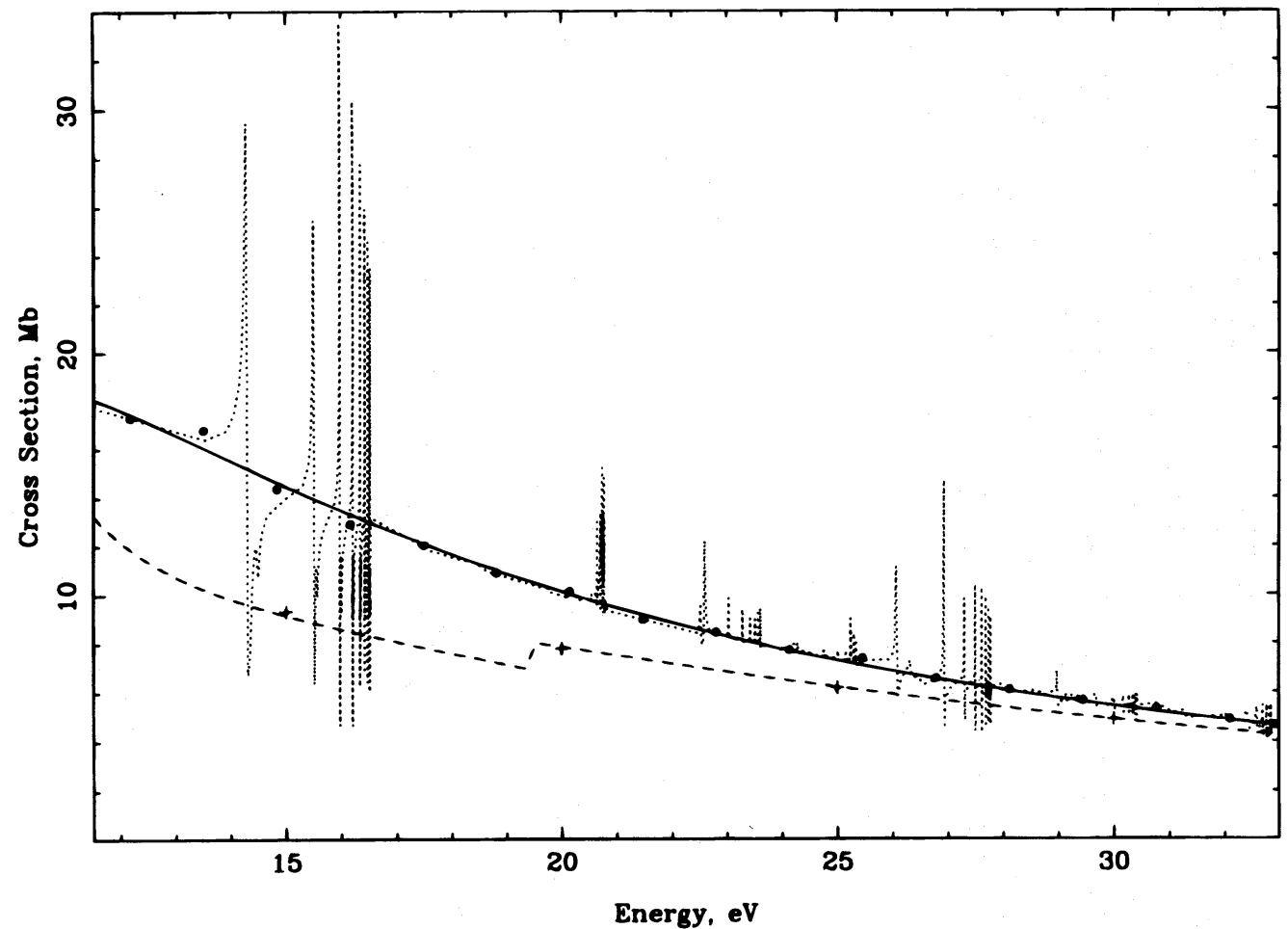

Fig. $2 a$

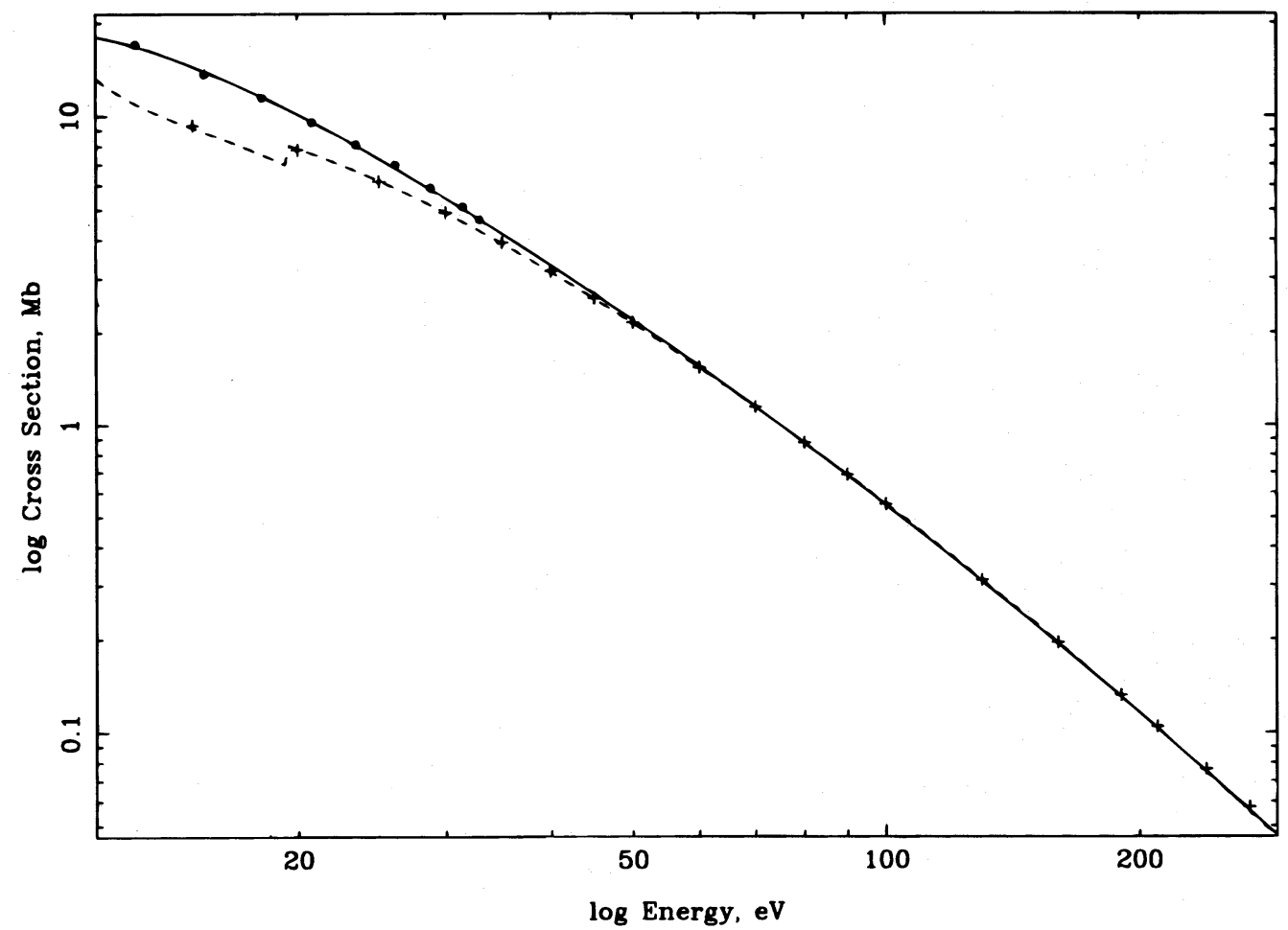

Fig. $2 b$

Fig. 2.-(a) Total photoionization cross sections of C I. Solid line, present work; dashed line, fit from Paper II; dotted line, Opacity Project data; circles, Opacity Project data interpolated and smoothed over resonances; crosses, calculations from Reilman \& Manson (1979). The cross sections are shown in linear scale for the Opacity Project energy range. (b) Same as (a), with the cross sections shown in logarithmic scale from the threshold up to the $1 s$ ionization edge.

fit parameters that determine, respectively, the position and energy width of the new dip. We do not introduce this improvement into the present results, since our "standard" formula (1) gives satisfactory accuracy even for S I. This generalization could be helpful in the future for fitting new cross sections.

All the fitting parameters and experimental ionization threshold energies $E_{\text {th }}$ taken from Kelly (1987) are listed in 


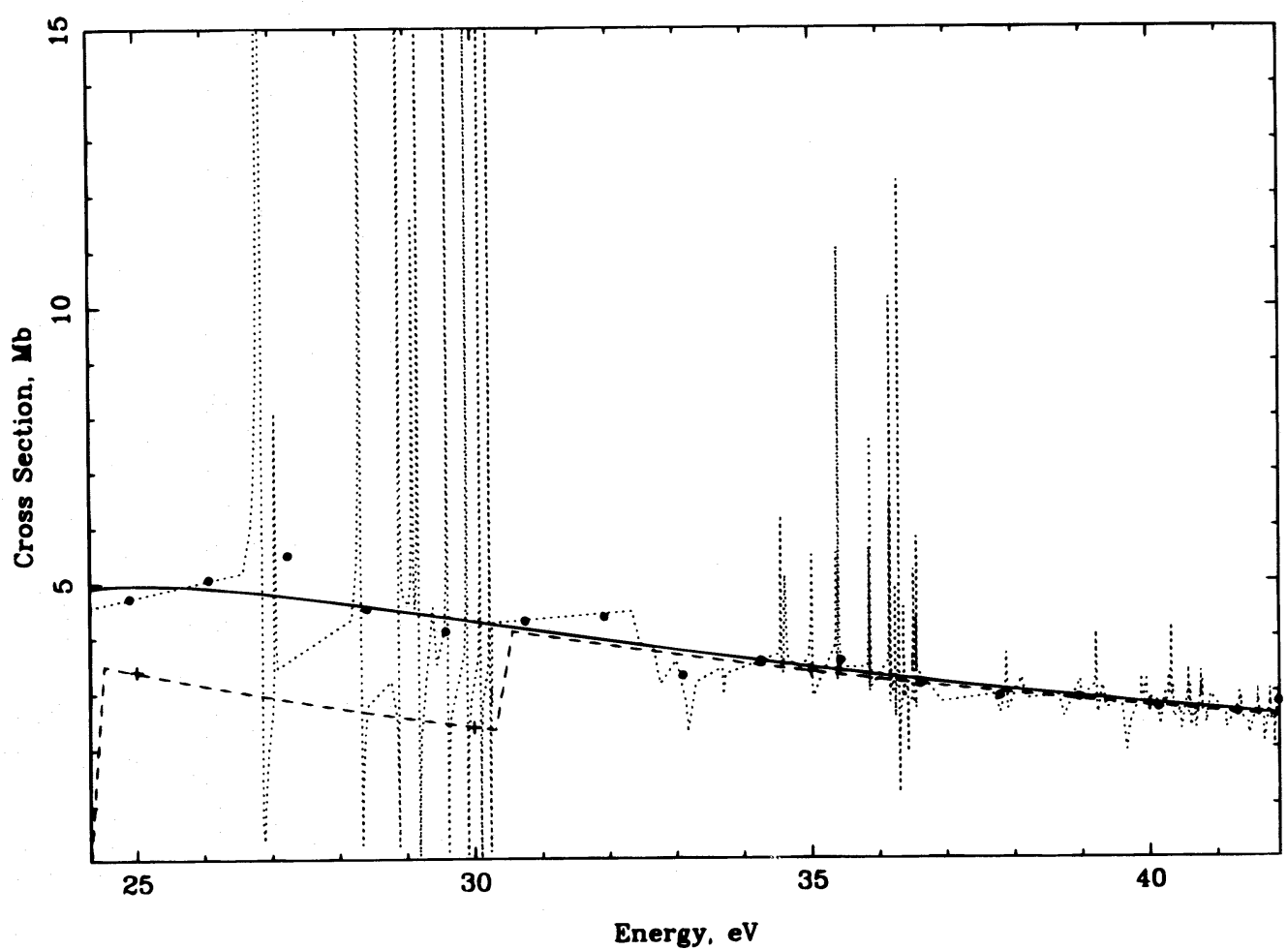

Fig. 3a

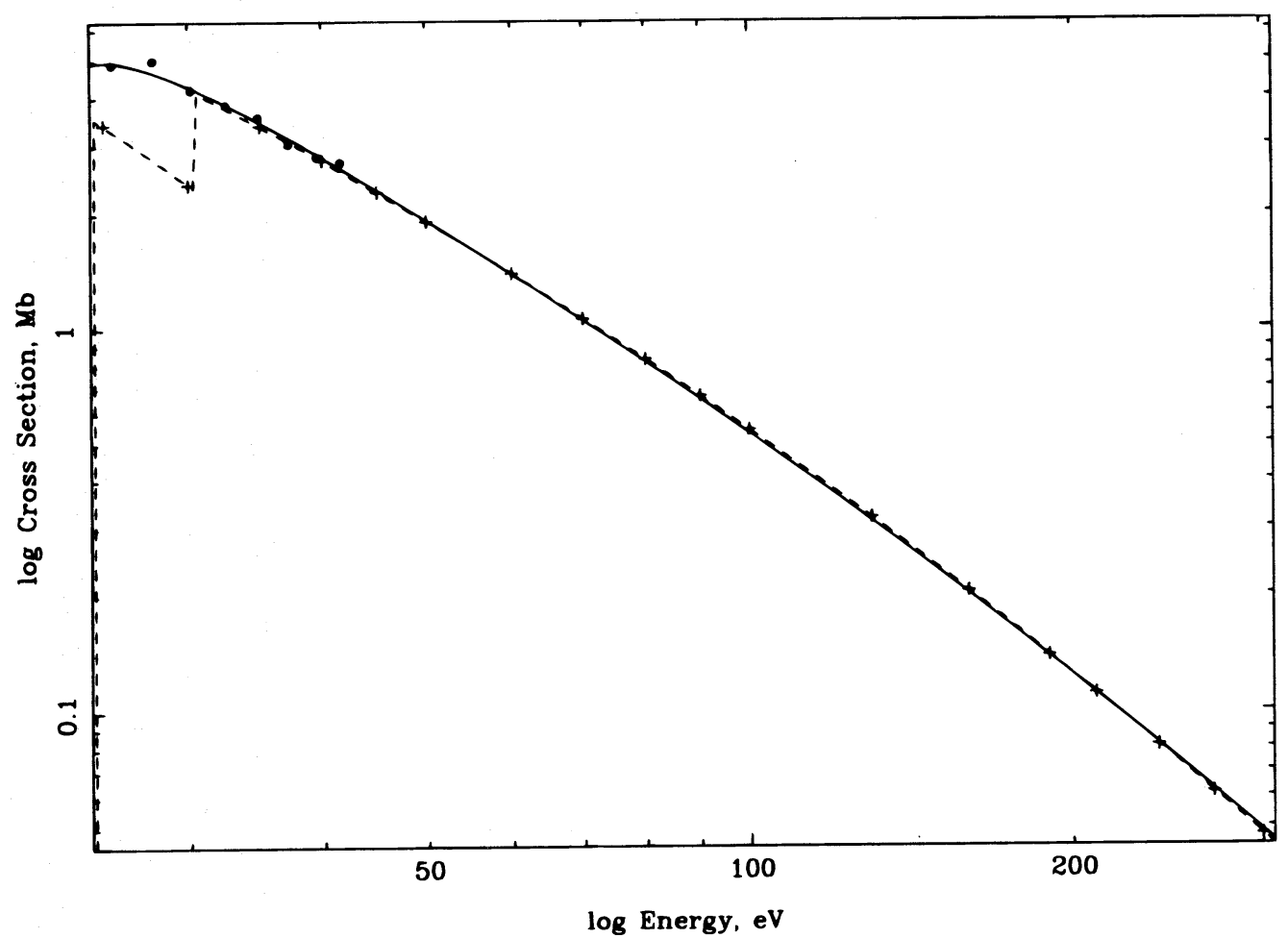

Fig. $3 b$

Fig. 3.-(a) Same as Fig. $2 a$ for C II. (b) Same as Fig. $2 b$ for C II.

Table 1. The fits are obtained for energies below the energy of the subsequent inner shell ionization edge. For species with $3 \leq N \leq 10$, this is the $1 s$ shell ionization energy; for $11 \leq N \leq 18$ this is the $2 p$ shell ionization energy; for $19 \leq N \leq 26$ this is the $3 p$ shell ionization energy. These energies, taken from Paper II, are listed as $E_{\max }$ in Table 1. At $E \geq E_{\max }$, the present fits should be supplemented by the fits to the inner shell photoionization cross sections given in Paper II. For H-like and He-like species $(N \leq 2)$, the only ground state shell is the $1 s$ shell. Since the present fitting 


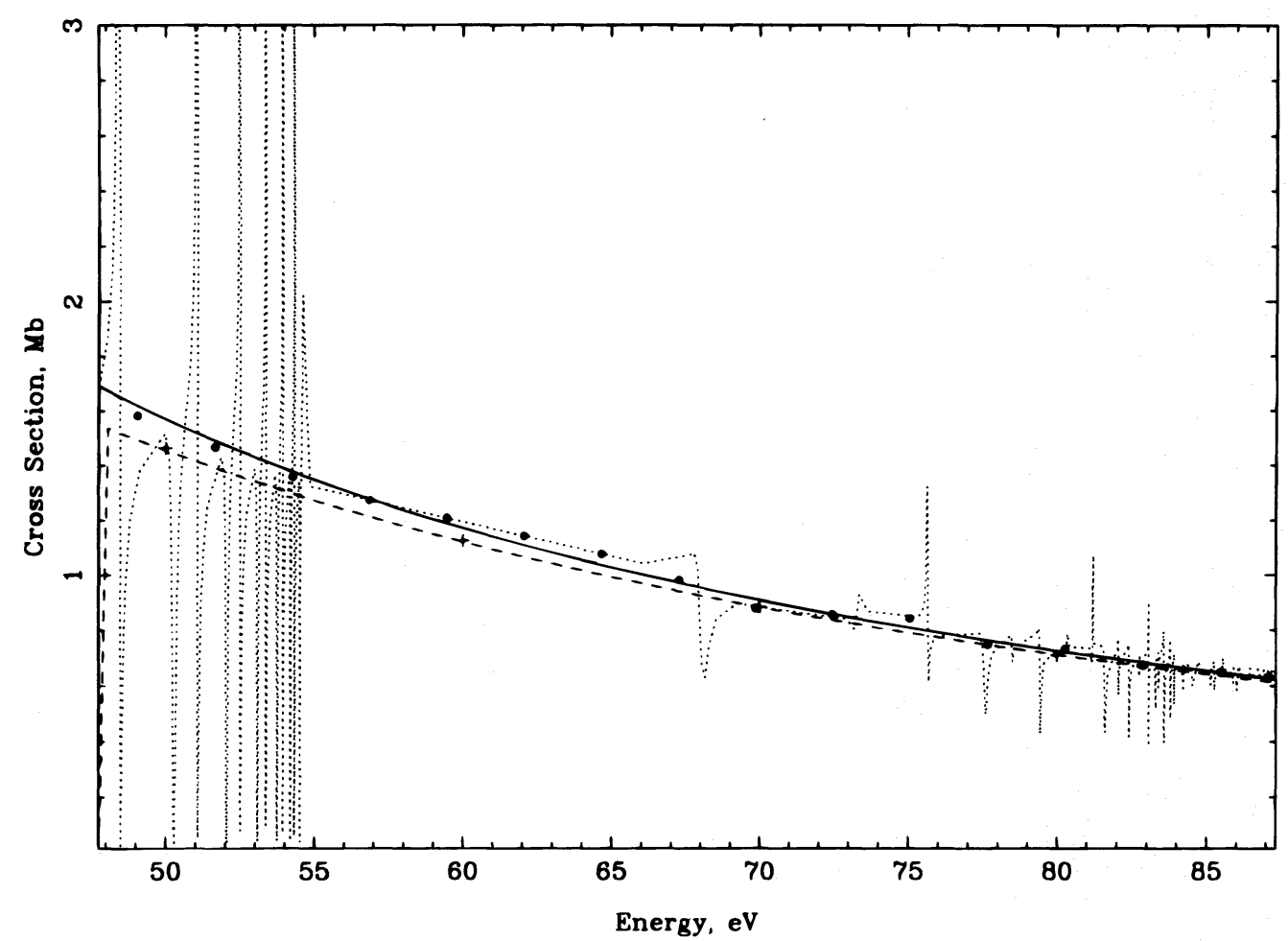

Fig. $4 a$

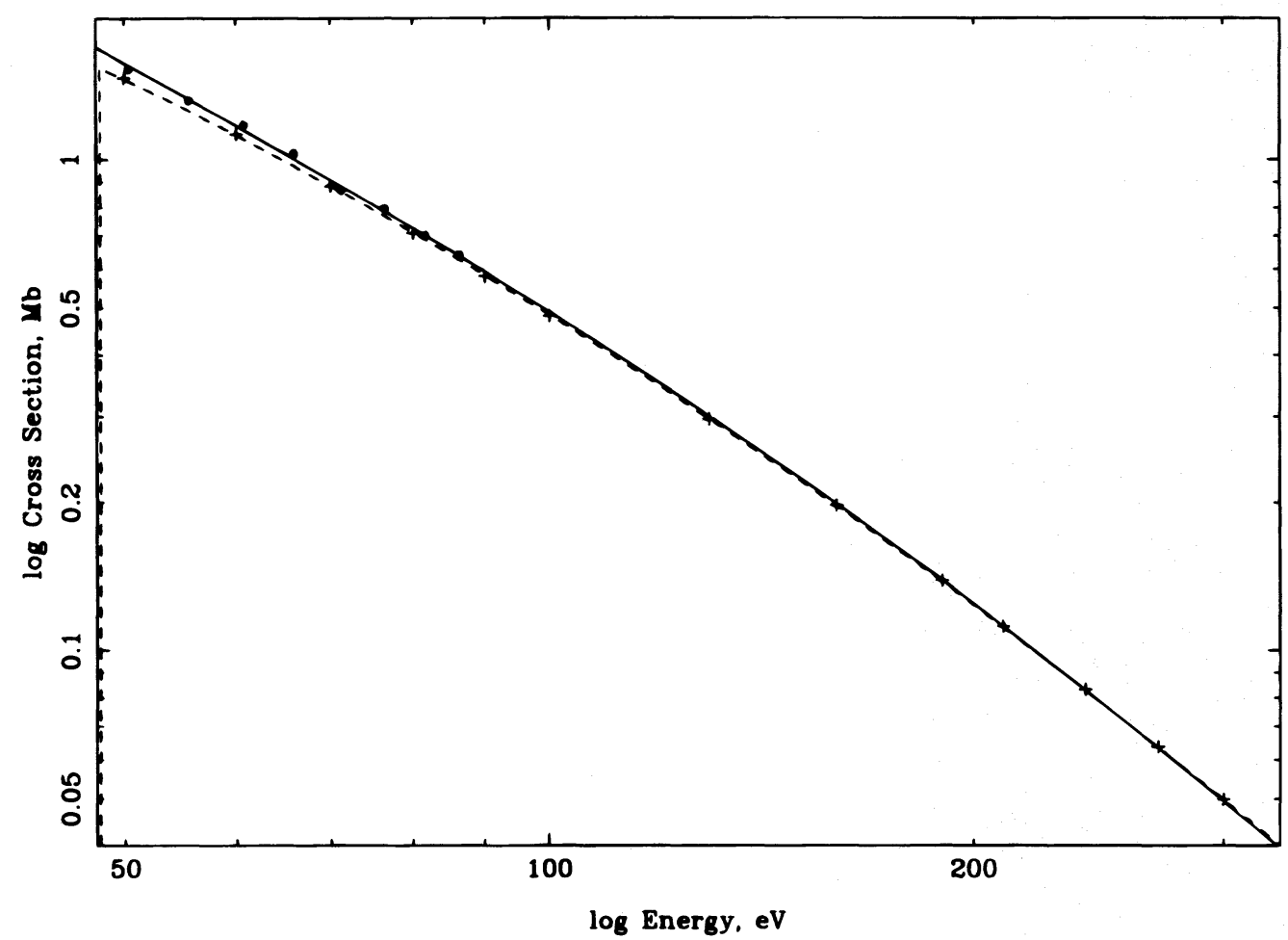

Fig. $4 b$

Fig. 4.-(a) Same as Fig. $2 a$ for C III. (b) Same as Fig. $2 b$ for C III.

formula has a correct nonrelativistic asymptote, our fits are accurate at all nonrelativistic energies. Thus, we put $E_{\max }=$ $50 \mathrm{keV}$ for the $\mathrm{H}$-like and He-like species. Note that for the $\mathrm{H}$-like, He-like (except $\mathrm{He}$ I), and Na-like (except $\mathrm{Na}$ I) species, which do not show resonances in the OP calculations, we keep the fits to the cross sections from Paper II.

All the fits can be divided to several classes of accuracy listed here, starting with the most accurate: 


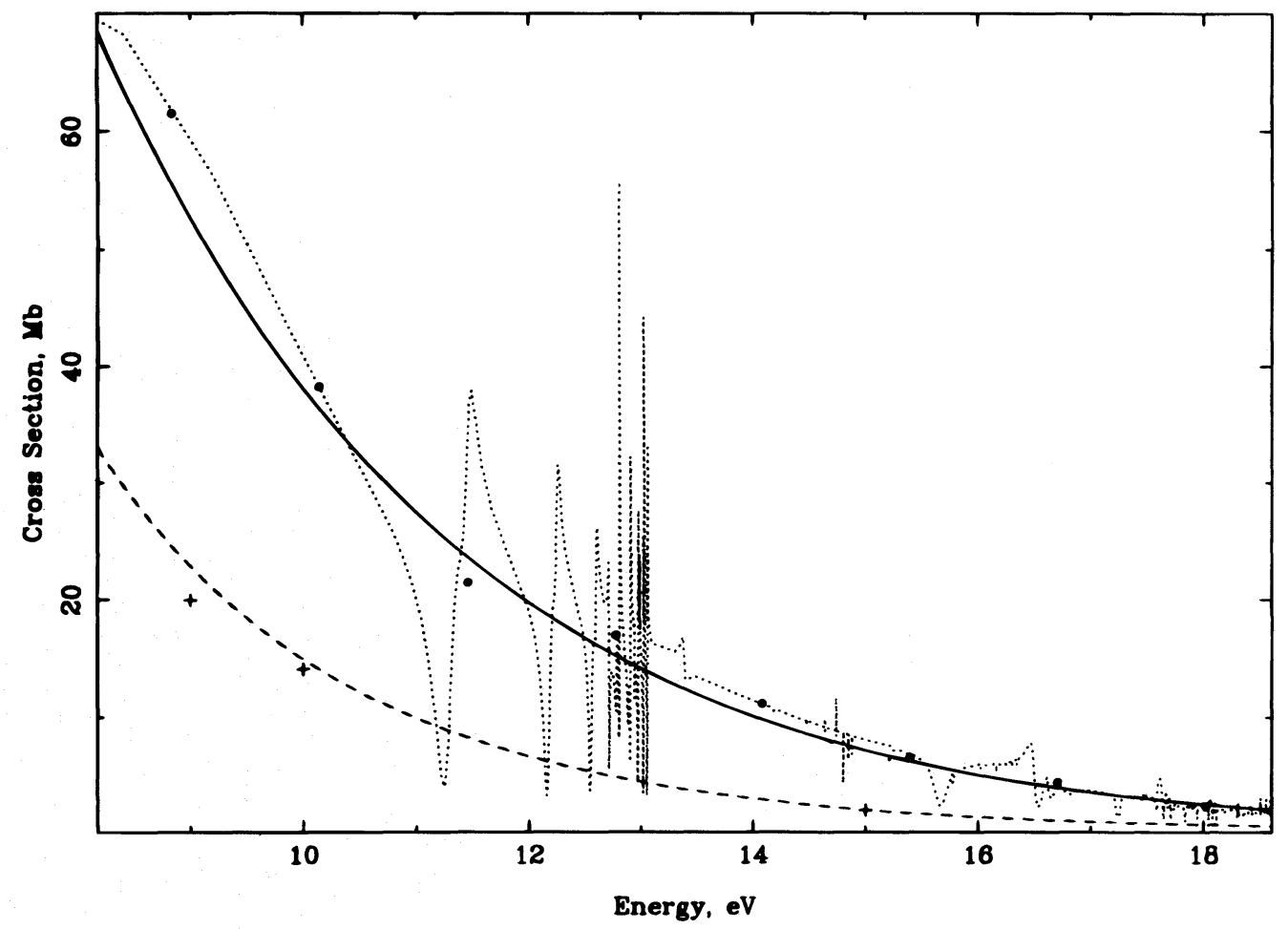

Fig. $5 a$

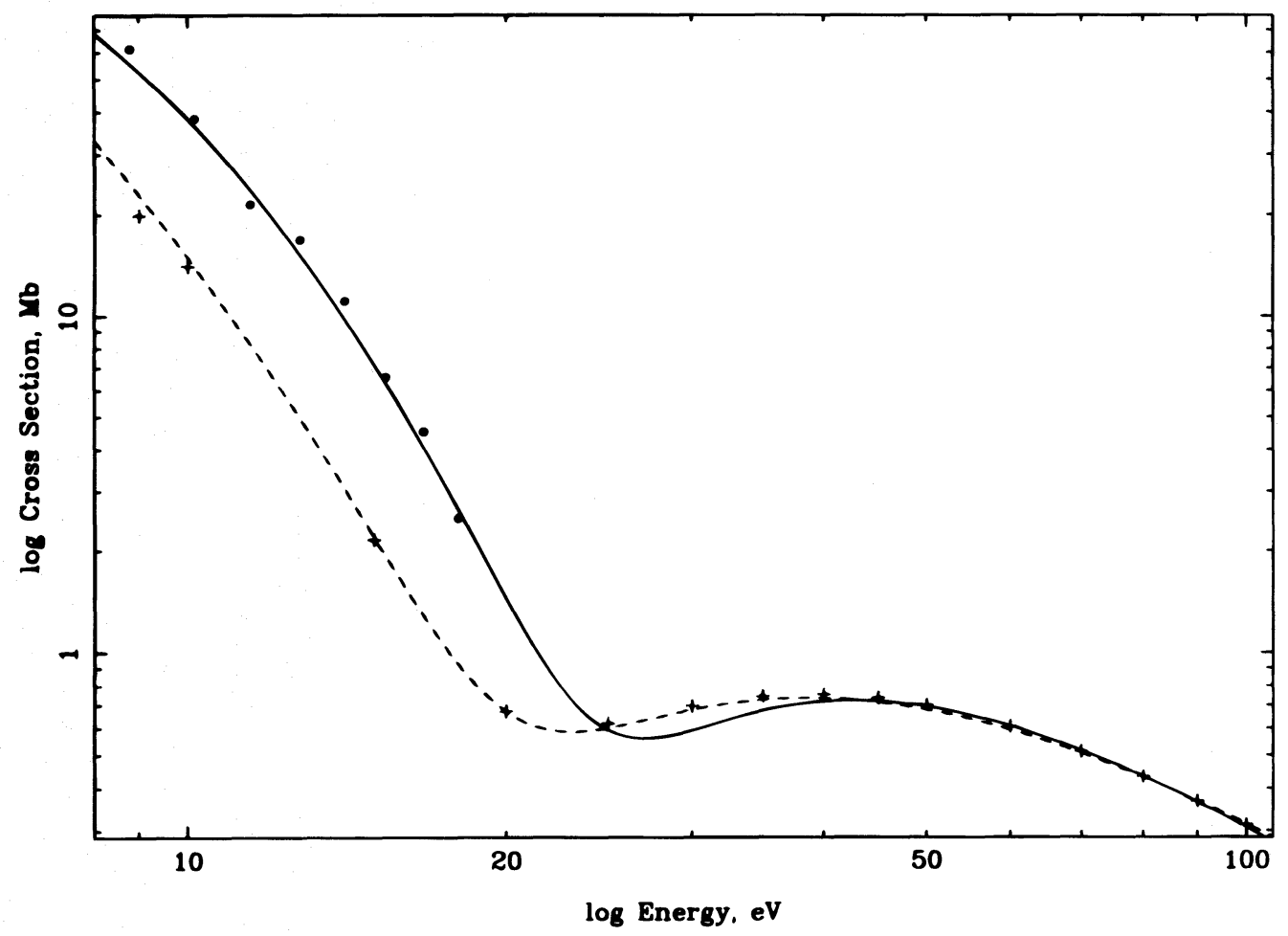

Fig. $5 b$

Fig. 5.-(a) Same as Fig. $2 a$ for Si I. (b) Same as Fig. $2 b$ for Si I. The cross sections are shown in logarithmic scale from the threshold up to the $2 p$ ionization edge.

A. Hydrogenic cross sections. They are known exactly, and rms accuracy of the fits is better than $0.2 \%$.

B. Cross sections without near-threshold resonances (Helike, Li-like, and Na-like species).
C. Regular cross sections. In these data, smoothed resonances reliably match the background cross section.

D. The same as $\mathrm{C}$, but for neutral and low-ionized species with $N \geq 13$. They are less accurate than cross sections of 


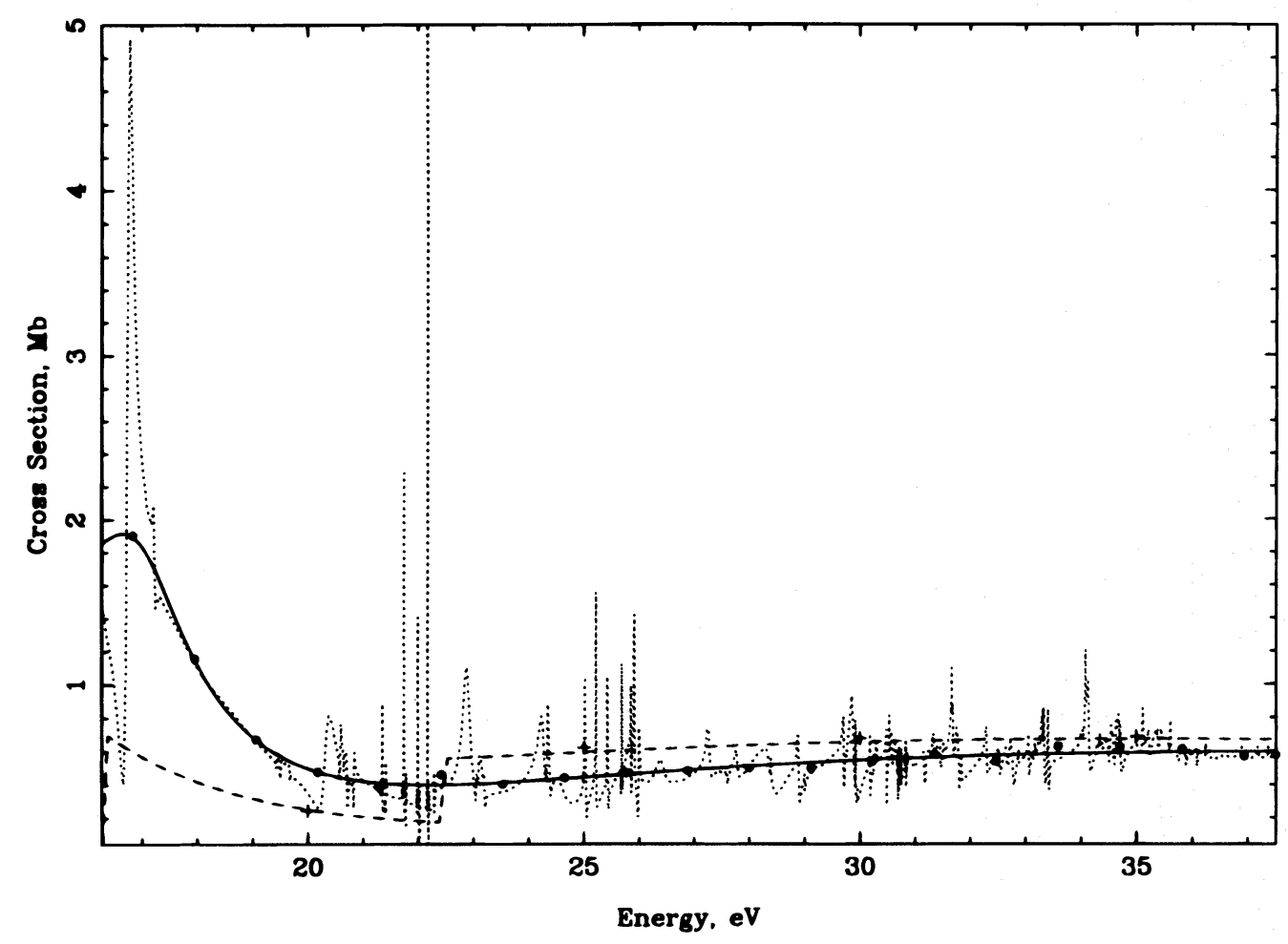

Fig. $6 a$

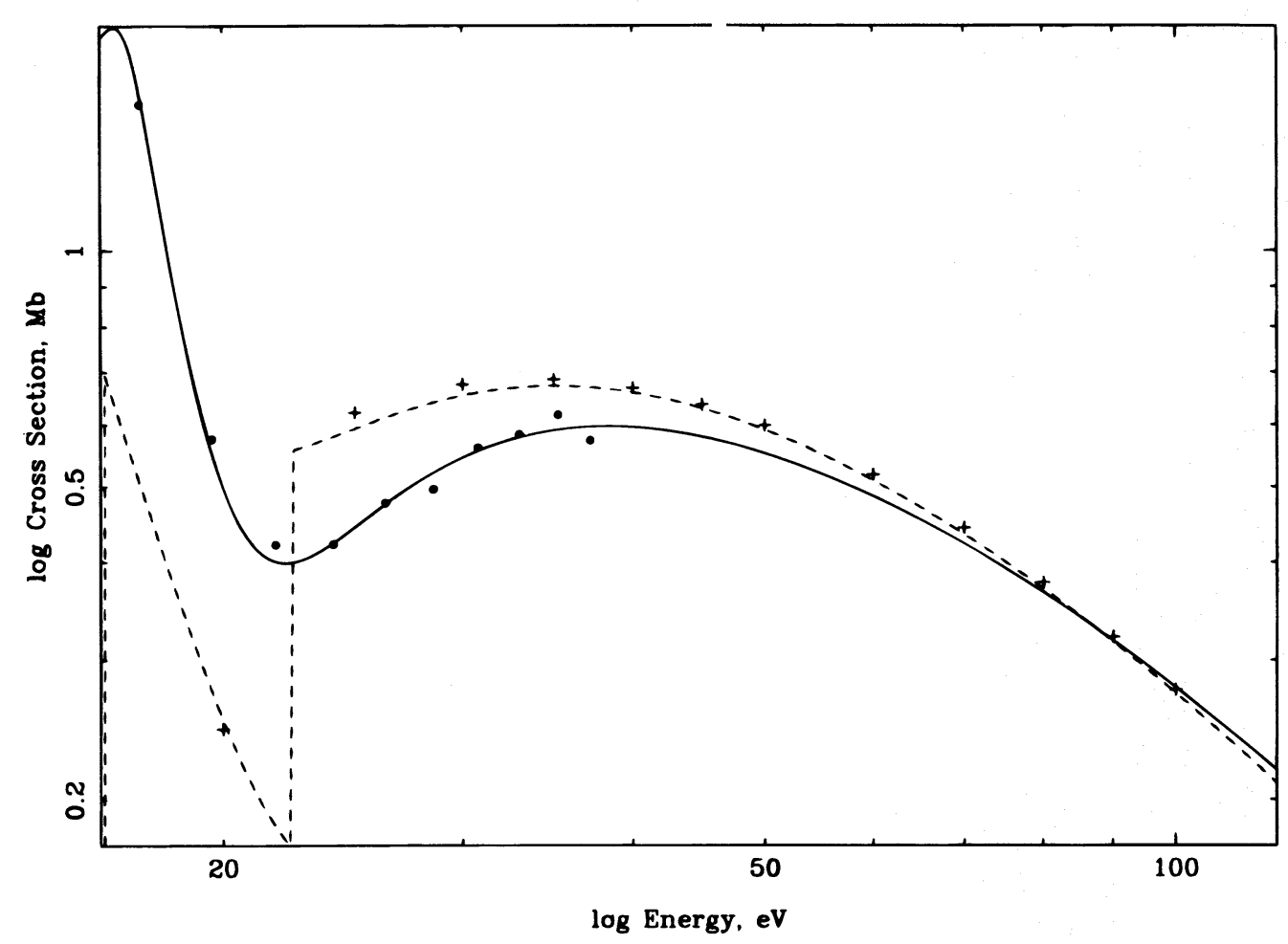

Fig. $6 b$

Fig. 6. - (a) Same as Fig. $2 a$ for Si II. (b) Same as Fig. $5 b$ for Si II.

class $\mathrm{C}$ (see discussion in $\S 2$ ).

E. Cross sections with broad near-threshold resonances.

F. The OP cross sections calculated with an insufficient number of target states in the close-coupling expansion.
Some comments concerning the fits to experimental cross sections deserve mention. The He I data by Samson et al. (1994) have the estimated accuracy better than $2 \%$ in lowenergy region, and better than $10 \%$ in high-energy region,

\section{( ) American Astronomical Society - Provided by the NASA Astrophysics Data System}




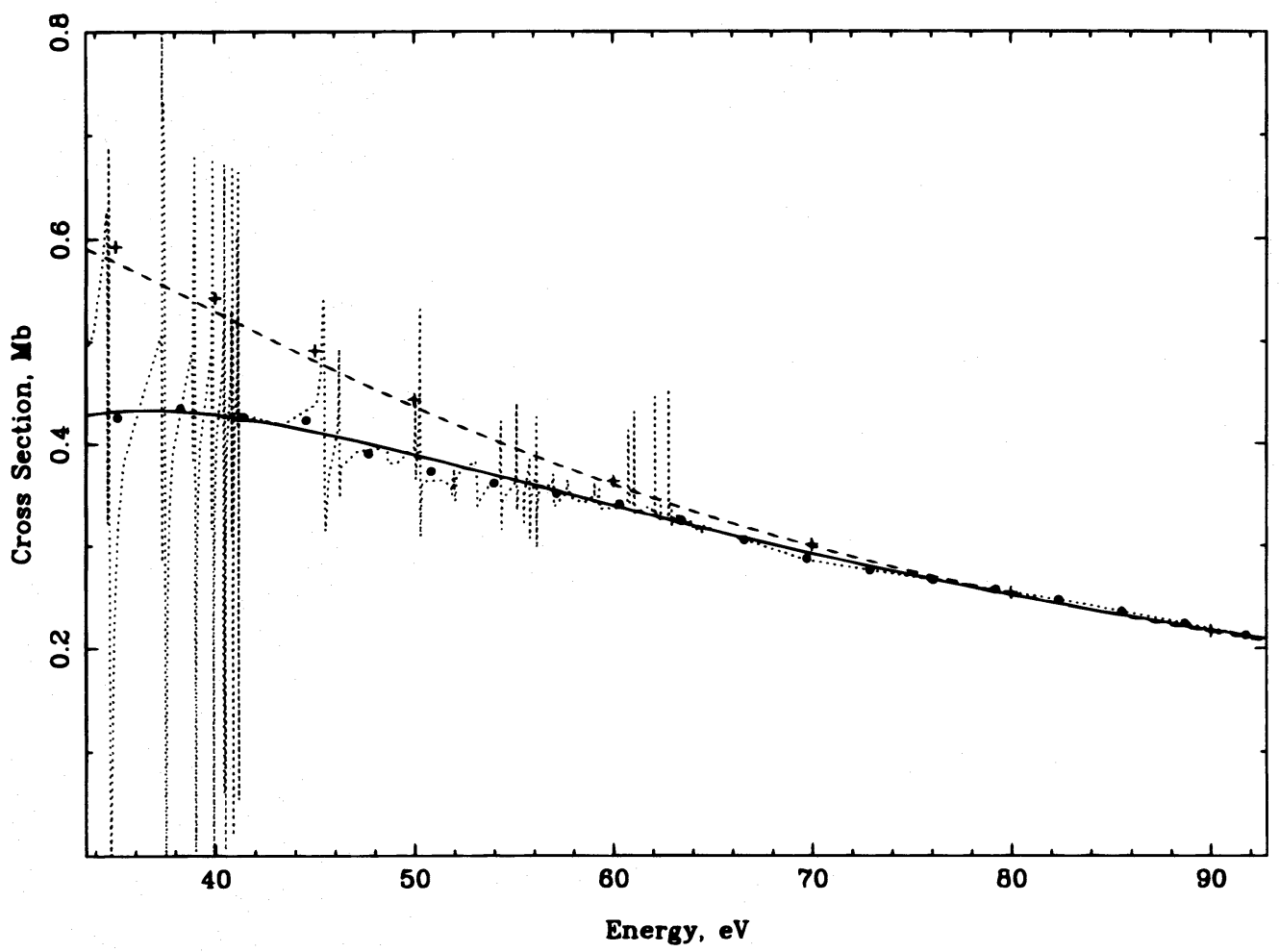

Fig. $7 a$

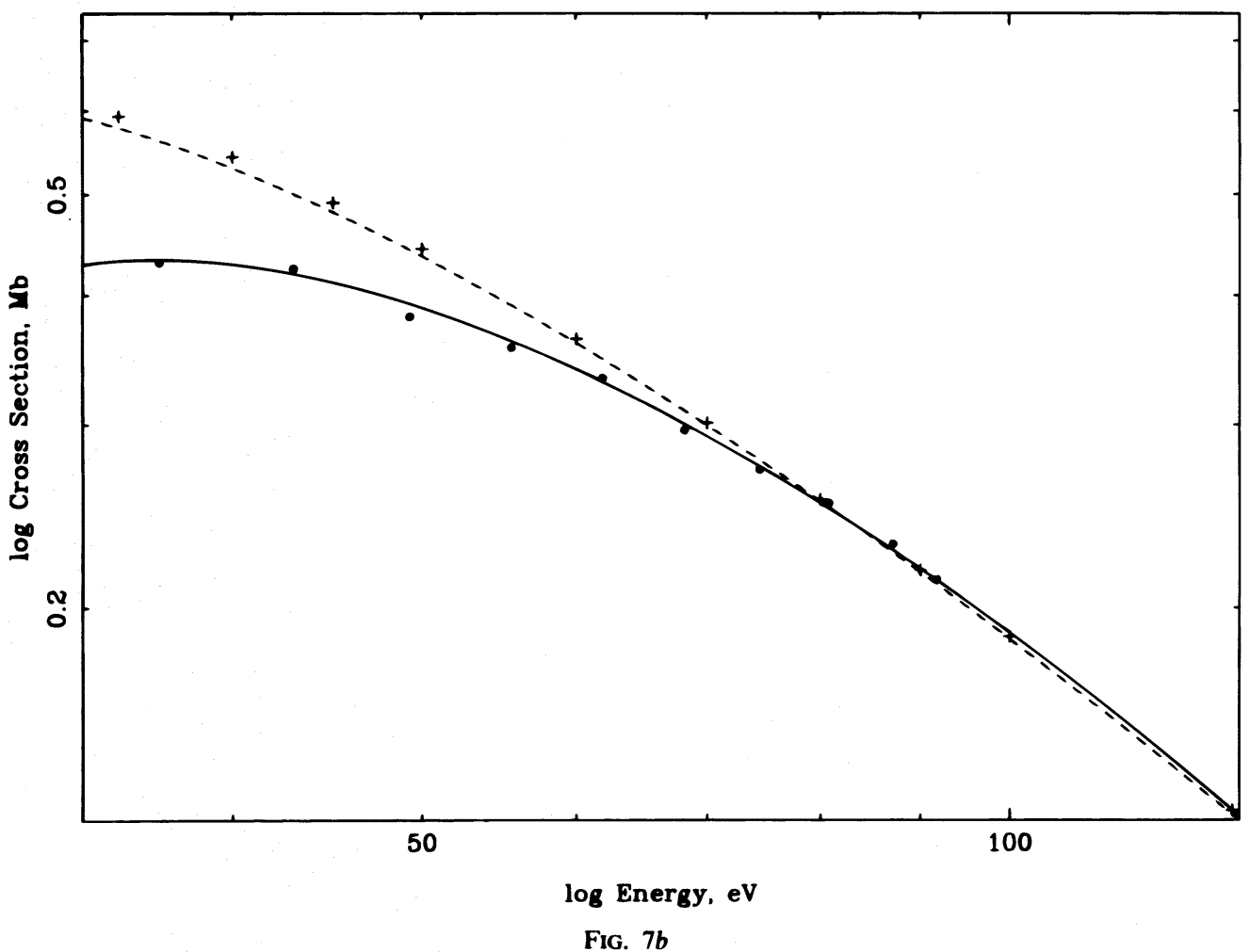

Fig. 7.-(a) Same as Fig. $2 a$ for Si III. (b) Same as Fig. $5 b$ for Si III.

and our fit does not lead to any loss of accuracy, keeping in mind that it matches the smoothed cross section in the region of resonances. The Ar I experimental data recommended by Marr \& West (1976) have lower accuracy (see
Samson et al. 1991) and contain uncertainties as high as $30 \%$. The Ca II experimental data by Lyon et al. (1987) include several huge resonances that cannot be smoothed at any reasonable energy step. 
As an illustration, Figures 2-7 compare the present fits with the OP and Reilman \& Manson (1979) data and also with the fits to the HDS cross sections from Paper II for the neutral, singly ionized and double ionized atoms of the astrophysically important elements $\mathrm{C}(Z=6)$ and $\mathrm{Si}$ $(Z=14)$. At high energies, the present fits coincide with those from Paper II.

In conclusion, the present fits are generally accurate at low energies, where they are based on the smoothed OP or experimental photoionization cross sections, and at high energies where the fits are based on the HDS photoionization cross sections. The fit parameters in electronic form and a Fortran subroutine that implements these fits and fits from the Paper II are available through anonymous ftp at asta.pa.uky.edu, cd dima/photo, or through the World Wide Web page "Atomic Data for Astrophysics": http://www.pa.uky.edu/ verner/atom.html. The fits will be updated as soon as new, more accurate photoionization cross sections become available.

This work was supported by grants from the National Science Foundation (AST 93-19034) and NASA (NAGW 3315). We are grateful to M. J. Seaton for useful discussions on the Opacity Project data. We are also grateful to the Opacity Project team for their efforts to make the data available before publication through the TOPbase database. We acknowledge the use of the TOPbase version 0.7 database installed by A. K. Pradhan at the Ohio State University. We thank the referee, Rolf Mewe, for suggestions that improved the presentation of the paper. We also thank D. Cohen for his helpful comments.

\section{APPENDIX}

Table 2 lists references to the Opacity Project photoionization cross sections.

TABLE 2

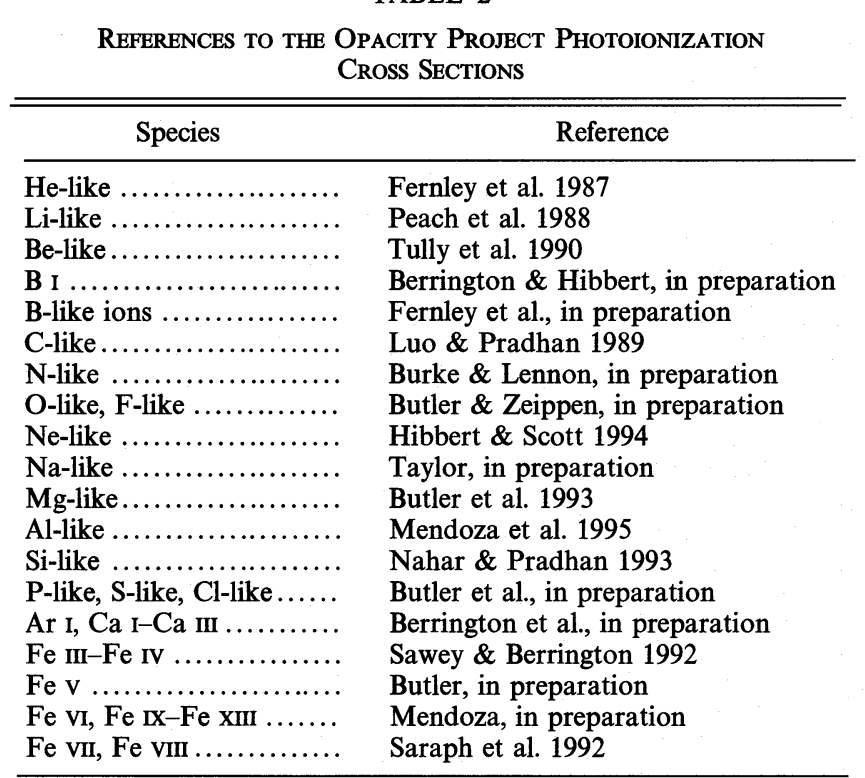

\section{REFERENCES}

Bautista, M. A., \& Pradhan, A. K. 1995, J. Phys. B, 28, L173

Butler, K., Mendoza, C., \& Zeippen, C. J. 1993, J. Phys. B, 26, 4409

Cunto, W., Mendoza, C., Ochsenbein, F., \& Zeippen, C.J. 1993, A\&A, 275 L5

Fernley, J. A., Taylor, K. T. \& Seaton, M. J. 1987, J. Phys. B, 20, 6457

Hibbert, A., \& Scott, M. P. 1994, J. Phys. B, 27, 1315

Kaastra, J. S., \& Mewe, R. 1993, A\&AS, 97, 443

Kelly, R. L. 1987, J. Phys. Chem. Ref. Data, 16, Suppl. 1

Luo, D., \& Pradhan, A. K. 1989, J. Phys. B, 22, 3377

Lyon, I. C., Peart, B., Dolder, K., \& West, J. B. 1987, J. Phys. B, 20, 1471

Marr, G. V., \& West, J. B. 1976, At. Data Nucl. Data Tables, 18, 497

Mendoza, C., Eissner, W., Le Dourneuf, M., \& Zeippen, C. J. 1995, J. Phys. B, 28,3485

Nahar, S. N., \& Pradhan, A. K. 1993, J. Phys. B, 26, 1109 1994, J. Phys. B, 27, 429
Peach, G., Saraph, H. E., \& Seaton, M. J. 1988, J. Phys. B, 21, 3669

Reilman, R. F., \& Manson, S. T. 1979, ApJS, 40, 815

Samson, J. A. R., \& Angel, G. C. 1990, Phys. Rev. A, 42, 1307

Samson, J. A. R., He, Z. X., Yin, L., \& Haddad, G. N. 1994, J. Phys. B, 27 887

Samson, J. A. R., Lyn, L., Haddad, G. N., \& Angel, G. C. 1991, J. de Phys. IV, 1, C1-99

Saraph, H. E., Storey, P. J., \& Taylor, K. T. 1992, J. Phys. B, 25, 4409

Sawey, P. M. J., \& Berrington, K. A. 1992, J. Phys. B, 25, 1451

Seaton, M. J., et al. 1992, Rev. Mex. Astron. Astrofis., 23, 19

Tully, J. A., Seaton, M. J., \& Berrington, K. A. 1990, J. Phys. B, 23, 3811

Verner, D. A., Barthel, P. D., \& Tytler, D. 1994, A\&AS, 108, 287

Verner, D. A., \& Yakovlev, D. G. 1995, A\&AS, 109, 125 (Paper II)

Verner, D. A., Yakovlev, D. G., Band, I. M., \& Trzhaskovskaya, M. B. 1993, At. Data Nucl. Data Tables, 55, 233 (Paper I) 